WU B 11-06

June, 222011

\title{
Transversity in hard exclusive electroproduction of pseudoscalar mesons
}

\author{
S.V. Goloskokov ${ }^{1}$ \\ Bogoliubov Laboratory of Theoretical Physics, Joint Institute for Nuclear \\ Research, \\ Dubna 141980, Moscow region, Russia \\ P. Kroll ${ }^{2}$ \\ Fachbereich Physik, Universität Wuppertal, D-42097 Wuppertal, Germany \\ and \\ Institut für Theoretische Physik, Universität Regensburg, \\ D-93040 Regensburg, Germany
}

\begin{abstract}
Estimates for electroproduction of pseudoscalar mesons at small values of skewness are presented. Cross sections and asymmetries for these processes are calculated within the handbag approach which is based on factorization in hard parton subprocesses and soft generalized parton distributions (GPDs). The latter are constructed from double distributions. Transversity GPDs are taken into account; they are accompanied by twist-3 meson wave functions. For most pseudoscalarmeson channels a combination of $\widetilde{H}_{T}$ and $E_{T}$ plays a particularly prominent role. This combination of GPDs which we constrain by moments obtained from lattice QCD, leads with the exception of the $\pi^{+}$and $\eta^{\prime}$ channels, to large transverse cross sections.
\end{abstract}

\footnotetext{
${ }^{1}$ Email: goloskkv@theor.jinr.ru

${ }^{2}$ Email: kroll@physik.uni-wuppertal.de
} 


\section{Introduction}

In a recent paper [1] we have investigated hard exclusive electroproduction of positively charged pions within the framework of the handbag approach in the kinematical range of small skewness, $\xi$, and small invariant momentum transfer, $-t$ but large photon virtualities, $Q^{2}$. As in our previous studies of vector-meson electroproduction $[2,3]$ the partonic subprocess is calculated within the modified perturbative approach [4] in which quark transverse degrees of freedom as well as Sudakov suppressions are taken into account. In other words the transverse size of the produced meson is not ignored as in the collinear (leading-twist) approach. On the other hand, the partons entering the subprocess are viewed as being emitted and reabsorbed by the nucleon collinearly to the nucleon momenta. The GPDs which embody the soft physics, are constructed with the help of double distributions. In [1] the sketched approach has been used to analyze the HERMES data on $\pi^{+}$electroproduction $[5,6,7]$. Besides the use of the modified perturbative approach this analysis differs in the following aspects from previous studies based on the collinear approximation (e.g. [8] - [11]):

- The full electromagnetic form factor of the pion, $F_{\pi}\left(Q^{2}\right)$, is taken into account (see also Ref. [12]) as it is measured by the $F_{\pi}$ collaboration [13] at Jefferson Lab in just the same process

- Besides the pion pole there is an extra contribution to the GPD $\widetilde{E}$.

- There are substantial contributions from transversely polarized pho-

tons. This is particularly obvious from the $\sin \phi_{s}$-moment, $A_{U T}^{\sin \phi_{s}}$, of the $\pi^{+}$cross section measured with a transversely polarized target [6]. As it is argued in [1] the contributions from transversely polarized photons can be calculated within the handbag approach as a twist-3 effect consisting of a twist-3 pion wave function and the leading-twist transversity GPD $H_{T}$.

Here, in this work we are going to extend the analysis performed in [1] to other pseudoscalar meson channels, namely $\pi^{0}, K$ and $\eta\left(\eta^{\prime}\right)$ production. For these processes there are no small-skewness data available as yet but they may be measured at the upgraded Jefferson Lab facility or by the Compass experiment. In so far we believe that estimates for various pseudoscalar meson channels are of interest and timely. While for $\pi^{+}$production only 
the isovector combinations of the GPDs, $F^{(3)}=F^{u}-F^{d}\left(F=\widetilde{H}, \widetilde{E}, H_{T}\right)$, contribute, different flavor combinations are relevant for the other channels. This necessitates a careful reexamination of the parameterizations of the GPDs with particular regard to those of the individual flavors. In some cases, as for instance for $\widetilde{E}$, we have to revise slightly the parameterizations proposed in [1]. In a recent lattice-QCD study [14] large moments of the transversity GPD combination

$$
\bar{E}_{T}=2 \widetilde{H}_{T}+E_{T}
$$

has been found. With regard to this result we will also examine the role of this GPD which has not been taken into account in [1], in order to find out whether or not it provides substantial effects in observables for meson electroproduction.

The plan of the paper is the following: In the next section we will sketch the handbag approach to meson electroproduction including twist-3 effects. In Sect. 3 we are going to present the parameterizations of the GPDs and compare them with recent results from lattice QCD in detail. Predictions for electroproduction of pseudoscalar mesons will be presented in the subsequent sections, for pions in Sec. 4 , for $\eta$ and $\eta^{\prime}$ in Sect. 5 and for kaons in Sect. 6. In Sect. 7 the asymmetries obtained with either a longitudinally polarized beam or target will be discussed. The paper is closed with a summary (Sect. 8).

\section{An outline of the handbag approach}

For details of the approach we are going to use it is referred to our previous papers, e.g. [1, 2, 3]. Here, we only sketch the basic facts. It is to be stressed that we consider the kinematical region of small $\xi$ and small $-t$ but large $Q^{2}$ and large photon-proton c.m.s. energy, $W$. Terms of order $(\sqrt{-t} / Q)^{n}$ $(n \geq 1)$ are neglected throughout.

The helicity amplitudes for electroproduction of pseudoscalar mesons, $\gamma^{*} p \rightarrow P B$, through longitudinally polarized photons read

$$
\begin{aligned}
& \mathcal{M}_{0+, 0+}^{P}=\sqrt{1-\xi^{2}} \frac{e_{0}}{Q}\left[\left\langle\widetilde{H}^{P}\right\rangle-\frac{\xi^{2}}{1-\xi^{2}}\left\langle\widetilde{E}_{\text {n.p. }}^{P}\right\rangle-\frac{\xi(m+M) Q^{2}}{1-\xi^{2}} \frac{\rho_{P}}{t-m_{P}^{2}}\right], \\
& \mathcal{M}_{0-, 0+}^{P}=\frac{e_{0}}{Q} \frac{\sqrt{-t^{\prime}}}{m+M}\left[\xi\left\langle\widetilde{E}_{\text {n.p. }}^{P}\right\rangle+(m+M) Q^{2} \frac{\rho_{P}}{t-m_{P}^{2}}\right] .
\end{aligned}
$$


Helicities are labeled by their signs or by zero. The usual abbreviation $t^{\prime}=$ $t-t_{0}$ is employed where [11]

$$
t_{0}=-2 \frac{\left(m^{2}+M^{2}\right) \xi^{2}+\left(M^{2}-m^{2}\right) \xi}{1-\xi^{2}}
$$

is the minimal value of $-t$ corresponding to forward scattering. The mass of the nucleon (meson, final state baryon) is denoted by $m\left(m_{P}, M\right), e_{0}$ is the positron charge and the skewness is related to Bjorken- $x$ by

$$
\xi=\frac{x_{\mathrm{Bj}}}{2-x_{\mathrm{Bj}}}\left[1+m_{P}^{2} / Q^{2}\right]
$$

The pole contribution, see Fig. 1, occuring in $\pi^{+}$and $K^{+}$production has the residue

$$
\rho_{P}=g_{P p B} F_{P p B}(t) F_{P}\left(Q^{2}\right),
$$

where $g_{P p B} F_{P p B}(t)$ is the coupling of the meson to the proton-baryon vertex and $F_{P}$ represents the electromagnetic form factor of the meson for which we use the experimental values. For $\pi^{0}$ and $\eta$ production $\rho_{P}$ is zero. Last not least the item $\left\langle F^{P}\right\rangle$ in (2) denotes a convolution of the GPD $F$ with an appropriate subprocess amplitude to be calculated from a set of Feynman graphs of which a typical leading-order example is shown in Fig. 1,

$$
\left\langle F^{P}\right\rangle=\sum_{\lambda} \int_{-1}^{1} d \bar{x} \mathcal{H}_{0 \lambda, 0 \lambda}^{P}\left(\bar{x}, \xi, Q^{2}, t=0\right) F^{P}(\bar{x}, \xi, t) .
$$

The label $\lambda$ refers to the unobserved helicities of the partons participating in the subprocess. Within the modified perturbative approach the subprocess amplitude reads

$$
\begin{aligned}
\mathcal{H}_{0 \lambda, 0 \lambda}^{P}= & \int d \tau d^{2} b \hat{\Psi}_{P}\left(\tau,-\mathbf{b}, \mu_{F}\right) \hat{\mathcal{F}}_{0 \lambda, 0 \lambda}^{P}\left(\bar{x}, \xi, \tau, Q^{2}, \mathbf{b}, \mu_{R}\right) \\
& \times \alpha_{\mathrm{s}}\left(\mu_{R}\right) \exp \left[-S\left(\tau, \mathbf{b}, Q^{2}, \mu_{F}, \mu_{R}\right)\right]
\end{aligned}
$$

in the impact parameter (b) space. For the Sudakov factor $S$, the choice of the renormalization $\left(\mu_{R}\right)$ and factorization $\left(\mu_{F}\right)$ scales as well as the hard scattering kernels $\mathcal{F}$ or their respective Fourier transforms $\hat{\mathcal{F}}$, we refer to Ref. [3]. The last item in (7) to be explained is $\hat{\Psi}_{P}(\tau,-\mathbf{b})$ which represents the Fourier transform of the momentum-space light-cone wave function for the meson ( $\tau$ is the momentum fraction of the quark that enters the meson, 

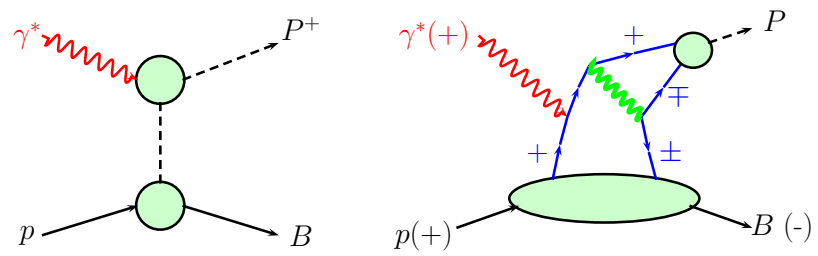

Figure 1: The pole contribution to the process $\gamma^{*} p \rightarrow P^{+} B$ (left) and the handbag graph (right) for electroproduction of pseudoscalar mesons. Helicities are specified for the amplitude $\mathcal{M}_{0-,++}$.

defined with respect to the meson momentum, the antiquark carries the fraction $1-\tau$ ).

As forced by the small $-t^{\prime}$ behavior of the HERMES data [6] on $A_{U T}^{\sin \phi_{s}}$ for $\pi^{+}$electroproduction one also needs the amplitude $\mathcal{M}_{0-,++}$. As is obvious from the parton helicities specified in the Feynman graph depicted in Fig. 1, the usual GPDs $\widetilde{H}$ and $\widetilde{E}$ which parameterize the nucleon matrix element for a situation where the emitted and reabsorbed partons have the same helicity, do not provide a contribution to $\mathcal{M}_{0-,++}$ with the required behavior $\mathcal{M}_{0-,++} \rightarrow$ const. for $t^{\prime} \rightarrow 0$. Angular momentum conservation forces this contribution to vanish in the forward direction. What is required, as has been discussed in detail in [1], is a contribution from the helicity-flip or transversity GPD $H_{T}[15,16]$ in combination with a twist-3 meson wave function

$$
\mathcal{M}_{0-,++}^{P}=e_{0} \sqrt{1-\xi^{2}} \int_{-1}^{1} d \bar{x} \mathcal{H}_{0-,++}^{P \text { twist }-3} H_{T}^{P} .
$$

This amplitude is parametrically suppressed by $\mu_{P} / Q$ with respect to the asymptotically leading amplitudes for longitudinally polarized photons. The parameter $\mu_{P}$ is large since it is enhanced by the chiral condensate

$$
\mu_{P}=\frac{m_{P}^{2}}{m_{1}+m_{2}}
$$

by means of the divergency of the axial-vector current. The $m_{i}$ are the current-quark masses of the meson's valence quarks. The parameter $\mu_{P}$ is scale dependent and evolves with the anomalous dimensions $4 / \beta_{0}=12 / 25$ for four flavors. For the $\eta$ and $\eta^{\prime}$ the situation is a bit more complicated. Decomposing these mesons into flavor-octet and single states, one has for the 
octet case

$$
\mu_{\eta_{8}}=3 \frac{m_{\eta_{8}}^{2}}{m_{u}+m_{d}+4 m_{s}} .
$$

The flavor-singlet $\eta$ is not related to the chiral condensate. In this case $\mu_{\eta_{1}}$ is just the $\eta_{1}$ mass which we approximate by that of the $\eta^{\prime}$. For pions, kaons and $\eta_{8}$ we take a value of $2 \mathrm{GeV}$ for the parameter $\mu_{p}$ at a scale of $2 \mathrm{GeV}$.

The twist-3 subprocess amplitude is given explicitly in [1] for $\pi^{+}$electroproduction. Its generalization to other pseudoscalar mesons is straightforward.

As we mentioned in the introduction we will also examine the role of the GPD (1) which contributes to the amplitude

$$
\mathcal{M}_{0+, \mu+}^{P}=-\frac{e_{0}}{2} \frac{\sqrt{-t^{\prime}}}{m+M} \int_{-1}^{1} d \bar{x} \mathcal{H}_{0-,++}^{P \text { twist-3 }} \bar{E}_{T}^{P},
$$

where $\mu= \pm 1$ indicates a transverse photon helicity. For our kinematical range of small $-t^{\prime}$ and small skewness contributions from the other transversity GPDs to the amplitudes (8) and (11) can be neglected. Note that $\widetilde{E}_{T}$ is an odd function of $\xi$ as a consequence of time-reversal invariance [16]. The double-flip amplitude $\mathcal{M}_{0_{-,-+}}$is also suppressed in the kinematical range of interest and neglected.

The pion-pole contributions to the amplitudes for transversely polarized photons are also taken into account. Explicit expressions for these contributions can be found in [1].

\section{The parameterization of the GPDs}

According to the discussion presented in Sect. 2 we need to model the GPDs $\widetilde{H}, \widetilde{E}, H_{T}$ and $\bar{E}_{T}$. It is to be emphasized that our parameterizations of the various GPDs are optimized for small skewness and small $-t$. We assume flavor-symmetric sea GPDs throughout. Hence, we have to model only the valence-quarks GPDs since the sea contributions cancel for $\pi^{+}$and $K^{+}$ production on this assumption. For the cases of $\pi^{0}$ and $\eta$ the sea does anyway not contribute. At present there is no experimental information from hard exclusive processes available which would allow for an examination of flavor-symmetry breaking effects in the sea. In recent studies of the polarized parton distributions $[17,18]$ however flavor-symmetry breaking effects in the

sea have been found. Thus, at least for $\widetilde{H}$ an estimate of the sea contribution 
on the basis of the double distribution ansatz, is possible. We found its sea contribution to the $\pi^{+}$production cross section to amount to about $1 \%$. It therefore can safely be neglected.

The valence-quarks GPDs are constructed with the help of their double distribution representation. For the latter the familiar ansatz [19]

$$
f_{i}^{a}(\rho, \eta, t)=\exp \left[\left(b_{i}-\alpha_{i}^{\prime} \ln \rho\right) t\right] F_{i}^{a}(\rho, \xi=t=0) \frac{3}{4} \frac{\left[(1-\rho)^{2}-\eta^{2}\right]}{(1-\rho)^{3}} \Theta(\rho)
$$

is made which consists of the forward limit, $F_{i}$, of the relevant GPD, a weight function and an exponential in $t$ with a profile function parameterized in a Regge-like fashion, i.e. with a slope of a Regge trajectory, $\alpha_{i}^{\prime}$, and a slope, $b_{i}$, of the residue function. The $t=0$ part of the Regge term is absorbed in the forward limit of the GPD which is given either by the polarized $\left(\Delta q^{a}(x)\right)$ or the transversity $\left(\delta^{a}(x)\right)$ parton distribution functions (PDFs) for quarks of flavor $a$ for the GPDs $\widetilde{H}^{a}$ or $H_{T}^{a}$. For the remaining two GPDs, $\widetilde{E}^{a}$ and $\bar{E}_{T}^{a}$, the forward limit is parameterized in a fashion analogously to the PDFs

$$
F_{i}^{a}(\rho, \xi=t=0)=N_{i}^{a} \rho^{-\alpha_{i}(0)}(1-\rho)^{\beta_{i}^{a}}
$$

with the parameters to be adjusted appropriately.

The full GPDs are obtained from the double distributions by the integral $[20,21]$

$$
F_{i}^{a}(\bar{x}, \xi, t)=\int_{-1}^{1} d \rho \int_{-1+|\rho|}^{1-|\rho|} d \eta \delta(\rho+\xi \eta-\bar{x}) f_{i}^{a}(\rho, \eta, t) .
$$

Evolution is neglected throughout since we are only interested in scales in the proximity of $2 \mathrm{GeV}$ which is the scale at which we quote the parameterizations of the GPDs. Below we will occasionally refer to moments of GPDs, in particular to moments at zero skewness. They are defined by

$$
F_{\text {in } 0}(t)=\int_{-1}^{1} d \bar{x} \bar{x}^{n-1} F_{i}(\bar{x}, \xi=0, t) .
$$

Before we turn to the discussion of the individual GPDs we want to comment on the Regge trajectories, $\alpha_{i}(t)$, used by us. They are to be understood as effective trajectories describing unspecified superpositions of poles and cuts. They are not related to the hadronic spectrum as for instance the prominent $\rho$ and $\omega$ Regge trajectories which contribute to the GPDs $H$ and 
$E$. The appearance of cuts in soft reactions dominated by unnatural-parity exchanges is a well-known fact. For definiteness let us consider the closely related reaction photoproduction of mesons $(M)$. For single-particle exchanges the corresponding helicity amplitudes satisfy the symmetry relation ${ }^{3}$

$$
\mathcal{M}_{-\mu^{\prime} \nu^{\prime},-\mu \nu}^{M}=\kappa \eta_{M}(-1)^{\mu-\mu^{\prime}} \mathcal{M}_{\mu^{\prime} \nu^{\prime}, \mu \nu}^{M},
$$

where, for vector (pseudoscalar) mesons, $\eta_{M}=+(-) 1$ and, for a (un)natural parity exchange, $\kappa=+(-) 1$. Eq. (16) relates for instance the amplitudes $\mathcal{M}_{0-,-+}$ and $\mathcal{M}_{0-,++}$. The first one is a double-flip amplitude which, by angular momentum conservation, is forced to vanish $\propto t^{\prime}$ for forward scattering. Therefore, $\mathcal{M}_{0-,++}$ also vanishes $\propto t^{\prime}$ for dynamical reasons although it is a non-flip amplitude. Hence, for $\pi^{+}$photoproduction, all single-particle exchanges vanish for forward scattering and would produce a forward dip in the differential cross section. This is in sharp contrast to experiment [22] the $\pi^{+}$photoproduction cross section exhibits a pronounced forward spike.

\section{$3.1 \widetilde{H}$}

The forward limit of $\widetilde{H}$, the polarized parton distribution ${ }^{4}$ is taken from [23]. The Regge parameters for $\widetilde{H}$ are quoted in Tab. 1. In [24] a profile function for $\widetilde{H}$ has been proposed that is more complicated than $b_{\tilde{H}}-\alpha_{\tilde{H}^{\prime}} \ln \rho$ in (12). It reads

$$
-\alpha_{\tilde{H}}^{\prime}(1-\rho)^{3} \ln \rho+b_{\tilde{H}}^{a}(1-\rho)^{3}+A_{\tilde{H}}^{a} \rho(1-\rho)^{2}
$$

In [24] the parameters appearing in (17), have been determined from the data on the axial-vector form factor [25] through the sum rule for $\widetilde{H}$ :

$$
\begin{array}{ll}
\alpha_{\tilde{H}}^{\prime}=0.9 \mathrm{GeV}^{-2}, & b_{\tilde{H}}^{u}=b_{\tilde{H}}^{d}=0.59 \mathrm{GeV}^{-2} \\
A_{\tilde{H}}^{u}=1.22 \mathrm{GeV}^{-2} & A_{\tilde{H}}^{d}=2.59 \mathrm{GeV}^{-2}
\end{array}
$$

This profile function is also valid at large $-t$. As has been noted in [24] there is a strong correlation between $\rho$ and $t$ in the zero-skewness GPDs: at large

\footnotetext{
${ }^{3}$ In $[1,3]$ it is shown that this relation also holds for the handbag amplitude at the twist2 level (with or without power corrections like $\mathbf{k}_{\perp}$ effects and to any order of perturbative QCD). The contributions from the GPDs $H$ and $E(\widetilde{H}$ and $\widetilde{E}$ ) corresponds to (un)natural parity exchange. Contributions from $\bar{E}_{T}$ behaves as natural parity exchanges while those from $H_{T}$ do not possess the property (16).

${ }^{4} \mathrm{~A}$ suitable parameterization of these PDFs can be found in [3]. As compared to that work the parameterization of $\Delta u_{v}$ is changed somewhat.
} 


\begin{tabular}{|c||c|c|c||c|c|}
\hline GPD & $\alpha(0)$ & $\alpha^{\prime}\left[\mathrm{GeV}^{-2}\right]$ & $b\left[\mathrm{GeV}^{-2}\right]$ & $N^{u}$ & $N^{d}$ \\
\hline$\widetilde{H}$ & 0.48 & 0.90 & 0.59 & - & - \\
$\widetilde{E}_{\text {n.p. }}$ & 0.48 & 0.45 & 0.9 & 14.0 & 4.0 \\
$H_{T}$ & -0.02 & 0.45 & 0 & 0.78 & -1.01 \\
$\bar{E}_{T}$ & 0.3 & 0.45 & 0.5 & 6.83 & 5.05 \\
\hline
\end{tabular}

Table 1: Regge parameters and normalizations of the GPDs, quoted at a scale of $2 \mathrm{GeV}$.

(small) $-t$ the large (small) $\rho$-region dominates. With regard to this fact one can view the profile function in (12) as the small $-t$ approximation of the more complicated profile function (17). Indeed both the profile functions for $\widetilde{H}$ lead to practically the same results for the axial-vector form factor and for pion electroproduction at small $-t$. In Fig. 2 the first moment of the isovector combination of $\widetilde{H}$ which represents the axial form factor of the nucleon

$$
F_{A}(t)=\int_{-1}^{1} d \bar{x} \widetilde{H}^{(3)}(\bar{x}, \xi, t),
$$

is shown and compared to the experimental data [25] and to results obtained from a recent lattice-QCD simulation [26]. Agreement between the moments evaluated from $\widetilde{H}$ and experiment is to be observed while the lattice result exhibits a flatter $t$ dependence although it corresponds with the others at small $-t$. This flat $t$ dependence is probably related to the fact that the lattice-QCD results are evaluated from heavy quarks (corresponding to a pion mass of $352 \mathrm{MeV}$ ), the extrapolation to the chiral limit is not yet possible in general. Similar observations can also be made for higher moments and for the moments of the isoscalar GPD combination. In passing we remark that an analogous comparison for the Dirac and Pauli form factors [24] (or higher moments of $H$ and $E$ ) reveals similar results. It is also to be stressed that, with regard to the heavy pion used in present lattice calculations, utmost caution is advisable in any comparison between lattice and phenomenological results. 

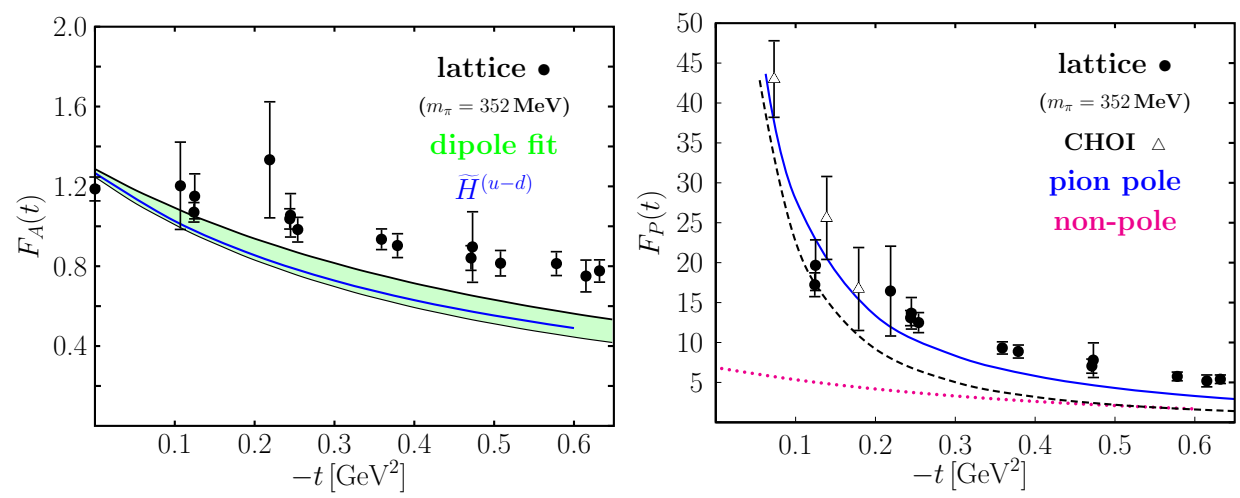

Figure 2: The axial (left) and the pseudoscalar (right) nucleon form factors versus $-t$. The solid circles represent the lattice results [26] for a pion mass of $352 \mathrm{MeV}$. The experimental data are taken from [25] (presented as a dipole fits with the errors shown as the green band) and from [27] (open triangles). The form factors evaluated from our GPDs are shown as solid lines. The dashed and dotted lines represent the pion-pole (with $\Lambda_{N}=0.44 \mathrm{GeV}$ ) and non-pole contributions to $F_{P}$, respectively. (Color online)

\section{$3.2 \widetilde{E}$}

As already mentioned this GPD consists of two parts, a contribution from the meson-pole and a non-pole term; the latter has been ignored in most studies (cf. for instance $[8,9,10,11]$ ). The pole term can be written as $[10]$

$$
\widetilde{E}_{p \rightarrow B}^{\text {pole }}=\Theta(|\bar{x}| \leq \xi) \frac{F_{P B}^{\text {pole }}(t)}{2 \xi} \Phi_{P}\left(\frac{\bar{x}+\xi}{2 \xi}\right)
$$

where $F_{P B}^{\text {pole }}$ is the pole contribution to the pseudoscalar form factor for the $p \rightarrow B$ transition ${ }^{5}$. The non-pole contribution, $\widetilde{E}_{\text {n.p. }}$, to $\widetilde{E}$ is parameterized as in (12) - (14). The parameters are quoted in Tab. 1 . The power $\beta_{\tilde{E}}^{u}=\beta_{\tilde{E}}^{d}$ is taken as 5 .

As a first check of our parameterization we evaluate the pseudoscalar form factor for the $p \rightarrow n$ transition, traditionally denoted by $F_{P}$

$$
F_{P}(t)=F_{P}^{\text {pole }}(t)+\int_{-1}^{1} d \bar{x} \widetilde{E}_{\text {n.p. }}^{(3)}(\bar{x}, \xi, t) .
$$

${ }^{5}$ The meson-exchange pole also contributes to the transversity GPDs $H_{T}, E_{T}$ and $\widetilde{E}_{T}$. The pole contribution to the amplitudes for transversely polarized photons are non-zero. 
With the help of PCAC and the Goldberger-Treiman relation the pion-pole contribution to the pseudoscalar form factor can be expressed by

$$
F_{P}^{\text {pole }}(t)=2 m f_{\pi} \frac{\sqrt{2} g_{\pi p n} F_{\pi p n}(t)}{m_{\pi}^{2}-t}
$$

where $f_{\pi}(=131 \mathrm{MeV})$ is the pion decay constant , $g_{\pi p n}(=13.1)$ the pionnucleon coupling constant [28] and

$$
F_{\pi p n}=\frac{\Lambda_{N}^{2}-m_{\pi}^{2}}{\Lambda_{N}^{2}-t} .
$$

The results for $F_{P}(t)$ are shown in Fig. 2 and compared to the experimental data [27] and results from lattice QCD [26]. One notes the same features as for the axial form factor - reasonable agreement at low $-t$ and a flatter $t$ dependence of the lattice results. The dominance of the pion pole at small $t$ is clearly visible.

From the analysis of $\pi^{+}$electroproduction [1] only the isovector combi-

nation of $\widetilde{E}_{\text {n.p. }}$ is fixed. In the absence of any experimental information that would allow to fix the normalization of the isoscalar combination too we have to rely on lattice-QCD results. Adjusting the normalizations to the second moments of $\widetilde{E}$ given in [26] which are free from the pion-pole contribution, we arrive at the values of the normalization constants for individual flavors quoted in Tab. 1. The lattice results [26] are however not accurate enough to allow for a determination of further differences in the parameterization (13) of $\widetilde{E}_{\text {n.p. }}$ for $u$ and $d$ quarks and, we repeat, are not extrapolated to the chiral limit.

\section{$3.3 \quad H_{T}$}

The forward limit of $H_{T}$, the transversity PDF, is taken from an updated version of the analysis of the data on the azimuthal asymmetry in semiinclusive deep inelastic scattering and in inclusive two-hadron production in electron-positron annihilation [29]. Since the parameters quoted in [29] differ from those obtained in a previous anlaysis which has been used by us in [1], our parameterization of $H_{T}$ is to be changed accordingly. Now, we parameterize the transversity PDFs as

$$
\delta^{a}=N_{H_{T}}^{a} \rho^{1 / 2}(1-\rho)\left[q^{a}(\rho)+\Delta q^{a}(\rho)\right] .
$$


This parameterization corresponds to a Regge intercept of about zero. The PDFs are taken from [23, 30]. The GPD is then calculated from (12) and (14) with the Regge parameters quoted in Tab. 1.

Moments of $H_{T}$ have been calculated in [31]. They are represented as dipole fits with extrapolations to the physical pion mass. In Tab. 2 the two lowest moments at $t=0$ are compiled and their $t$ dependence is shown and compared to the moments evaluated from our GPDs in Fig. 3. Our moments at $t=0$ which are practically those obtained by Anselmino et al [29], are about $40 \%$ smaller than the lattice results. As for the other GPDs our moments possess a somewhat steeper gradient than the lattice moments as, at least for $u$ quarks, can be seen from Fig. 3. The first moment of $H_{T}$, termed the tensor form factor,

$$
g_{T}^{a}(t)=\int_{-1}^{1} d \bar{x} H_{T}^{a}(\bar{x}, \xi, t),
$$

has also been calculated within a chiral quark-soliton model [32]. At $t=0$ the following values have been obtained in [32]

$$
H_{T 10}^{u}(0)=0.876, \quad H_{T 10}^{d}(0)=-0.251,
$$

which are close the lattice results, and therefore larger than our ones, too. On the other hand, the $t$ dependence obtained within that chiral quark-soliton model is even steeper than we found, see Fig. 3. Several other models (cf. [29] and references therein) also predict larger moments of the transversity PDFs than those obtained from the parameterization (24) which is based on the analysis performed in [29].

\section{$3.4 \bar{E}_{T}$}

The GPD $\bar{E}_{T}$ defined in (1), is a new element in our analysis, it has been ignored in [1]. According to lattice QCD [14] $\bar{E}_{T}$ has however large moments with the same sign and similar size for $u$ and $d$ quarks. It therefore seems to be expedient to examine its impact on electroproduction of pseudoscalar mesons.

The GPD $\bar{E}_{T}$ is parameterized as the other ones, (12) - (14), but in contrast to the situation for the other GPDs, its parameters can only be fixed with the help of lattice results. The latter are presented in [14] in form of a $p$-pole fit

$$
\bar{E}_{T n 0}^{a}(t)=\frac{\bar{E}_{T n 0}^{a}(0)}{\left[1-t /\left(p m_{a n}^{2}\right)^{2}\right]^{p}}
$$




\begin{tabular}{|l|c|c||c|c|c|}
\hline & {$[31]$} & this work & & {$[14]$} & this work \\
\hline$H_{T 10}^{u}$ & $0.857(13)$ & 0.585 & $\bar{E}_{T 10}^{u}$ & $2.93(13)$ & 2.93 \\
$H_{T 20}^{u}$ & $0.268(6)$ & 0.123 & $\bar{E}_{T 20}^{u}$ & $0.420(31)$ & 0.360 \\
$H_{T 10}^{d}$ & $-0.212(5)$ & -0.153 & $\bar{E}_{T 10}^{d}$ & $1.90(9)$ & 1.90 \\
$H_{T 20}^{d}$ & $-0.052(2)$ & -0.021 & $\bar{E}_{T 20}^{d}$ & $0.260(23)$ & 0.199 \\
\hline
\end{tabular}

Table 2: The first two moments of the transversity PDFs $H_{T}$ and $\bar{E}_{T}$ at $t=0$ quoted at the scale $2 \mathrm{GeV}$.

with $p=2.5$. The forward values, quoted in Tab. 2, are extrapolated to the chiral limit but not the mass parameters. The lowest moments at $t=0$ are the analogues of the familiar anomalous magnetic moments, $\kappa^{a}$, and are therefore termed tensor anomalous magnetic moments, $\kappa_{T}^{a}$. The lattice results for them are of the same magnitude as the usual ones but in contrast to those they are both positive. This is in agreement with model studies performed in [33] which also support the expectation that $\kappa_{T}^{a} \gtrsim\left|\kappa^{a}\right|$. The lattice result is further corroborated by large- $N_{c}$ considerations [33] which provide $\kappa_{T}^{u} \approx \kappa_{T}^{d}$ and by a recent constituent quark model [34]. It has been speculated [33] that $\bar{E}_{T}$ is linearly related to the Boer-Mulders function, $h_{1}^{\perp a}$, [35] while the Sivers function, $f_{1 \top}^{a}$, is analogously related to the transversity PDFs. In a recent analysis [36] of the $\langle\cos (2 \phi)\rangle$ measurements [37, 38] some evidence has been obtained that the signs of the Boer-Mulders and the Sivers functions exhibit the same pattern as those of the lowest moments of $\bar{E}_{T}$ and $H_{T}$ at $t=0$ which are quoted in Tab. 2.

The parameters of $\bar{E}_{T}$ are fixed by us in such a way that the lowest moments from lattice QCD at $t=0$ are exactly reproduced and the second moments at least approximately. The $t$ dependence is assumed to be somewhat steeper than the lattice results as is the case for the other GPDs. This can be achieved with the parameters

$$
\beta_{E_{T}}^{u}=4, \quad \beta_{E_{T}}^{d}=5 .
$$

and those quoted in Tab. 1. The moments at $t=0$ are given in Tab. 2 and their $t$ dependencies are shown in Fig. 3. 

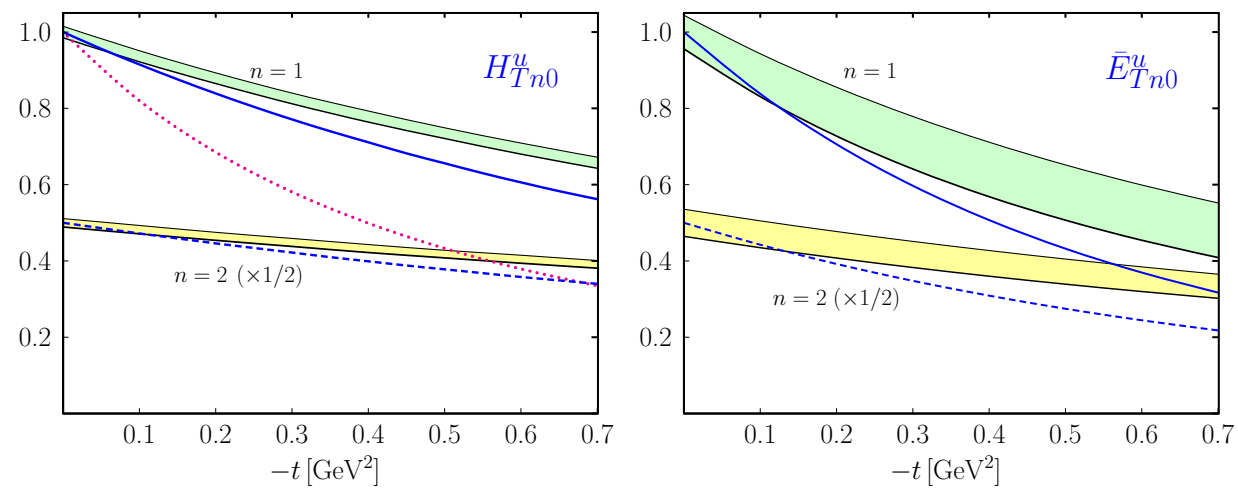

Figure 3: The first and second moments of $H_{T}$ (left) and $\bar{E}_{T}$ (right) for $u$ quarks scaled by the forward value. The results evaluated from our GPDs are shown as solid and dashed lines, the lattice-QCD results [14, 31] as bands indicating their uncertainties. The dotted line represents the chiral quarksoliton model [32]. (Color online)

We have checked that our GPDs respect various positivity bounds. Thus, for instance,

$$
\left(E \pm \bar{E}_{T}\right)^{2}(t=0) \leq 4 m^{2}(H-\widetilde{H}) \frac{\partial}{\partial t}\left(H+\widetilde{H} \pm 2 H_{T}\right)(t=0),
$$

and similar ones derived in [39] at zero skewness or bounds on $\widetilde{H}$ and $\widetilde{E}$ in terms of PDFs [40] as for instance

$$
\begin{aligned}
\left|\frac{\sqrt{-t^{\prime}}}{2 m} \xi \widetilde{E}^{q}\right| & \leq \frac{1}{4}\left[\sqrt{(q+\Delta q+2 \delta)_{x}(q-\Delta q)_{x^{\prime}}}\right. \\
& +\sqrt{(q-\Delta q)_{x}(q+\Delta q+2 \delta)_{x^{\prime}}} \\
& +\sqrt{(q+\Delta q-2 \delta)_{x}(q-\Delta q)_{x^{\prime}}} \\
& \left.+\sqrt{(q-\Delta q)_{x}(q+\Delta q-2 \delta)_{x^{\prime}}}\right]
\end{aligned}
$$

where the GPDs are to be taken at $\bar{x}, \xi$ and $t$ while the PDFs are either to be evaluated at $x$ or $x^{\prime}$, the individual momentum fractions of the emitted or reabsorbed partons,

$$
x=\frac{\bar{x}+\xi}{1+\xi}, \quad x^{\prime}=\frac{\bar{x}-\xi}{1-\xi} .
$$




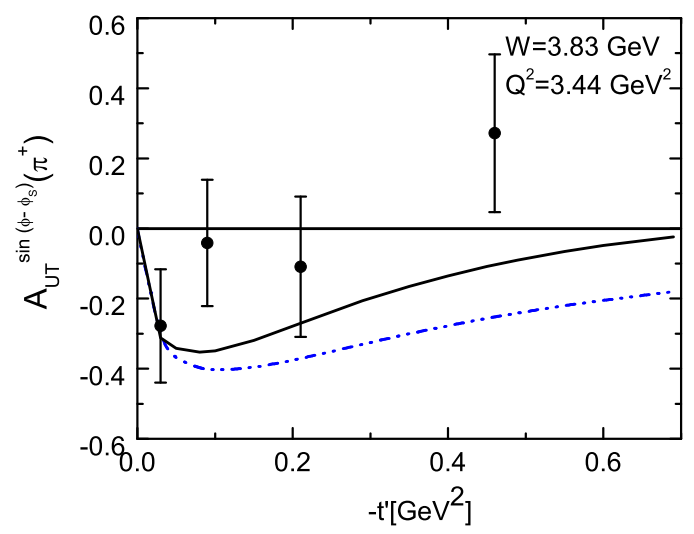

Figure 4: Results for the $\sin \left(\phi-\phi_{s}\right)$ moment for $\pi^{+}$production shown as solid line. The dash-dot-dotted line is obtained under neglect of $\bar{E}_{T}$. Data are taken from [6]. (Color online)

All bounds hold for a given flavor; (30) only holds in the DGLAP region. Flavor labels are omitted for convenience in (30).

\section{Electroproduction of pions}

Since we have slightly changed the parameterizations of the GPDs as compared to [1] and have taken into account $\bar{E}_{T}$ now, we have to repeat the computation of $\pi^{+}$electroproduction in order to examine the quality of the new results. As a matter of fact, it turns out that they are very close to the previous results presented in [1]. Their agreement with experiment $[5,6,7]$ is reasonable. The contribution from $\bar{E}_{T}$ to the cross section is tiny, much smaller than the errors of the HERMES cross section data [5], since the flavor combination $\bar{E}_{T}^{u}-\bar{E}_{T}^{d}$, occuring in $\pi^{+}$electroproduction, is small. It is only noticeable in the asymmetry parameters where it improves the agreement with experiment in general. As an example the $\sin \left(\phi-\phi_{s}\right)$ moment of the cross section measured with a transversely polarized target [6] is shown in Fig. 4 ( $\phi$ is the azimuthal angle between the lepton and the hadron plane while $\phi_{s}$ specifies the orientation of the target spin vector with respect to the lepton plane).

As a second example the unseparated cross section as well as its longi- 

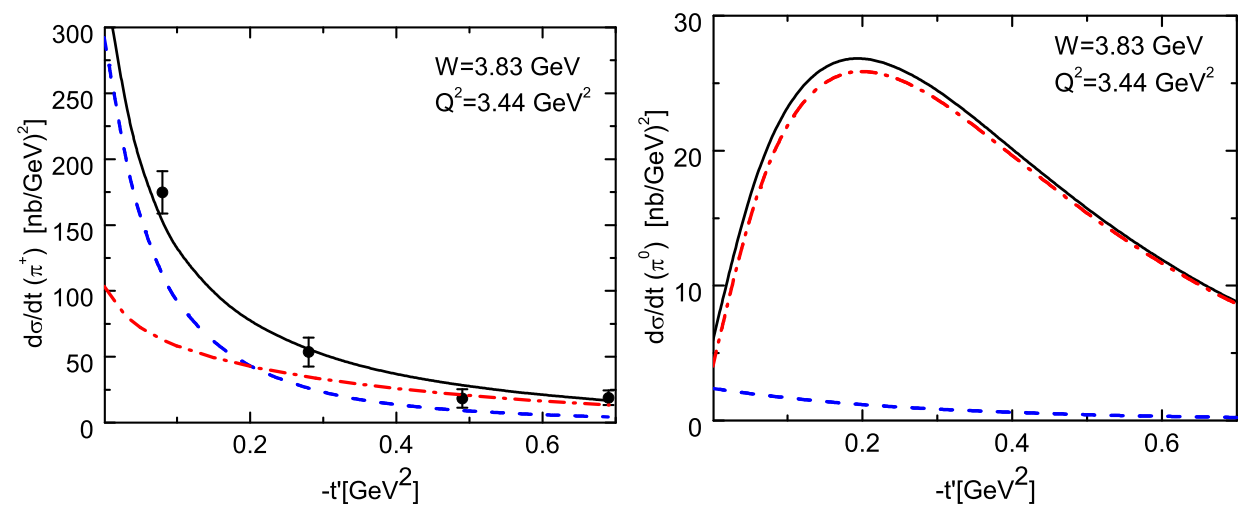

Figure 5: Results for the $\pi^{+}$(left) and $\pi^{0}$ (right) cross sections. The solid (dashed, dash-dotted) lines represent the unseparated (longitudinal, transverse) cross sections. The $\pi^{+}$data are taken from [5]. (Color online)

tudinal and transverse components $\left(d \sigma=d \sigma_{T}+\varepsilon d \sigma_{L}\right)$ for $\pi^{+}$production are shown in Fig. 5 at $Q^{2}=3.44 \mathrm{GeV}^{2}$ and $W=3.83 \mathrm{GeV}$. For the ratio of the longitudinal and transverse photon fluxes, $\varepsilon$, a value of 0.8 is taken. Evidently, the agreement with the HERMES data [5] is very good. The longitudinal cross section is dominant at low $-t^{\prime}$ but if $-t^{\prime}$ becomes larger than about $0.2 \mathrm{GeV}^{2}$ the transverse cross section takes the lead. A crossing of the two cross sections is also seen in the large-skewness Jefferson Lab data measured by the $F_{\pi}$ collaboration [13] although it occurs at a smaller value of $-t^{\prime}$. Actually the crossing takes place at about the same value of $t$ for our results and the $F_{\pi}$ data. It is to be stressed that the dominance of the longitudinal cross section at small $-t^{\prime}$ is a consequence of the strong pion pole. Without it one would have $d \sigma_{T}>d \sigma_{L}$ at all $t^{\prime}$. The pure pole contribution to the longitudinal cross section behaves as $-t /\left(t-m_{\pi}^{2}\right)^{2}$ at small $-t$. This factor has a pronounced maximum at $t=-m_{\pi}^{2}$. For $\xi \geq m_{\pi} /(2 m)$ the maximum lies outside the scattering region. Hence, the longitudinal cross section decreases continuously. On the other hand, for $\xi<m_{\pi} /(2 m)$ the above factor generates a maximum of $d \sigma_{L} / d t$ at a small value of $-t^{\prime}$. The maximum is visible in the cross section if $Q^{2} \lesssim\left(m_{\pi} / m\right) W^{2}$.

Before we turn to the discussion of $\pi^{0}$ production it is in order to comment on the pion wave functions which are needed in the calculation of the subprocess amplitudes (cf. (7)). In fact we use the same wave functions as 
in [1], namely a Gaussian in $\mathbf{k}_{\perp}$ with an associated distribution amplitudes $\Phi_{A S}=6 \tau(1-\tau)$ for the twist-2 subprocess amplitudes and $\Phi_{p} \equiv 1$ for twist 3. For the transverse-size parameter in the Gaussian we use $a_{\pi}=\left[\sqrt{8} \pi f_{\pi}\right]^{-1}$ $\left(=0.861 \mathrm{GeV}^{-1}\right)$ for twist 2 and $a_{\pi p}=1.8 \mathrm{GeV}^{-1}$ for twist 3 . There is an extra factor $\mathbf{k}_{\perp}$ multiplying the Gaussian for the twist-3 wave function. For a detailed discussion of such a $\mathbf{k}_{\perp}$ factor and its interpretation see [41, 42]. Higher-order Gegenbauer terms in the distribution amplitudes are strongly suppressed in the modified perturbative approach: The Sudakov factor in conjunction with the subprocess amplitude provides a series of power suppressed terms which are generated in the region of soft quark momenta $(\tau, 1-\tau \rightarrow 1)$. They grow with the Gegenbauer index and reduce the perturbative contribution. With increasing $Q^{2}$ the higher Gegenbauer terms become gradually more important (cf. the discussion in [43]). Since we are merely interested in rather small values of $Q^{2}$ the asymptotic distribution amplitude suffices for pion production.

Now, for $\pi^{0}$ electroproduction there is no contribution from the pion pole and the GPDs appear in the flavor combination

$$
\left(e_{u} F_{i}^{u}-e_{d} F_{i}^{d}\right) / \sqrt{2}
$$

in contrast to $F_{i}^{u}-F_{i}^{d}$ for $\pi^{+}$production ${ }^{6}$. From the relative signs and sizes of the GPDs for $u$ and $d$ quarks it is evident that the contributions from $\widetilde{H}$ and $H_{T}$ are large for $\pi^{+}$but small for $\pi^{0}$ production while for $\widetilde{E}_{\text {n.p. }}$ and $\bar{E}_{T}$ the situation is reversed. The contributions from the latter two GPDs are small for $\pi^{+}$but large for $\pi^{0}$ production. As a consequence the longitudinal $\pi^{0}$ cross section is much smaller than the corresponding $\pi^{+}$one even if the pionpole contribution is subtracted. Another consequence is that $\pi^{0}$ production is dominated by contributions from transversely polarized photons which are mainly generated by $\bar{E}_{T}$. The latter contribution is by order of magnitude stronger in $\pi^{0}$ than in $\pi^{+}$production where a strong cancellation between $\bar{E}_{T}^{u}$ and $\bar{E}_{T}^{d}$ occurs. The importance of $\bar{E}_{T}$ in $\pi^{0}$ production is obvious from the forward dip in the cross section (see Fig. 5); it only contributes to helicity flip amplitudes, see (11).

As we repeatedly mentioned our GPDs are optimized for the range of small skewness. Therefore, our approach cannot readily be applied at the kinematics accessible at the Jefferson Lab. Ignoring this supposition and ap-

\footnotetext{
${ }^{6}$ For the production of $\pi^{+}$and kaons the quark charges are absorbed in the hard scattering amplitude.
} 


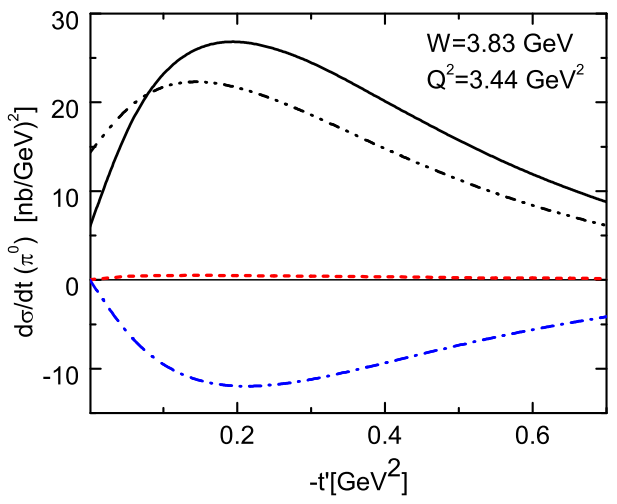

Figure 6: Unseparated and partial cross sections for $\pi^{0}$ production. The solid (dashed, dash-dotted) line represents the unseparated (longitudinaltransverse, transverse-transverse) cross section. The dash-dot-dotted line is evaluated from the modified GPDs, see text. (Color online)

plying our approach in its present form at Jefferson Lab kinematics straightforwardly, one runs into difficulties with $\rho^{0}$ production - the cross section is at variance with experiment [44] by order of magnitude [2, 45]. For $\phi$ production [46], on the other hand, our approach works quite well. The reason for this failure of the handbag approach with $\rho^{0}$ production is not understood but it is likely be related to the valence quarks. Similar difficulties may happen for electroproduction of pions. With this admonition in mind one may compare our approach with the preliminary CLAS data on $\pi^{0}$ production [47] (at $W \simeq 2.5 \mathrm{GeV}$ and $Q^{2} \simeq 2.3 \mathrm{GeV}^{2}$ ). It turns out that the magnitude of the cross section is about right. Even a little forward dip is seen in the CLAS data on the cross section. This effect has also been observed in a Hall A experiment [48]. It is important to realize that the depth of the dip is determined by the ratio of the amplitudes $\mathcal{M}_{0-,++}$ and $\mathcal{M}_{0+,++}$, i.e. it is influenced by the relative strength of $H_{T}$ and $\bar{E}_{T}$. We remind the reader that the normalization of the first GPD is fixed by the transversity PDF determined in [29] while the second one is constrained by lattice-QCD results [14]. A change of these GPDs may lead to a different shape of the cross section in the forward region. An example of such a modification of the GPDs is also shown in Fig. 6 and compared to the result evaluated from the standard parameterization of the GPDs presented in Sect. 3. For this variant we modify 

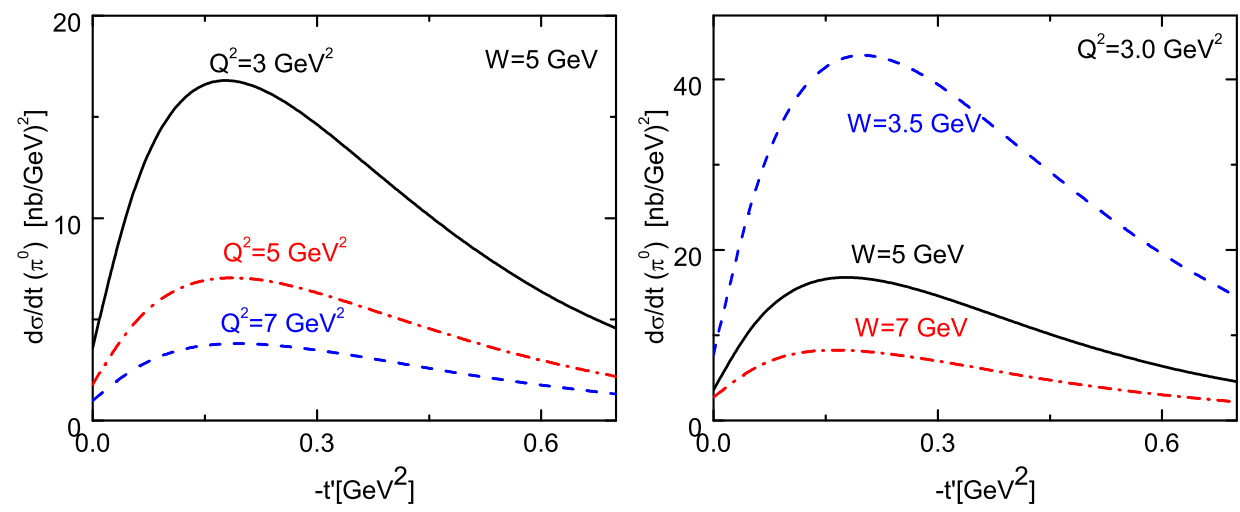

Figure 7: Results for $\pi^{0}$ the cross section versus $t^{\prime}$ at $W=5 \mathrm{GeV}$ for sample values of $Q^{2}$ (left) and at $Q^{2}=3 \mathrm{GeV}^{2}$ for various values of $W$ (right). The polarization of the photon flux is taken as $\varepsilon=0.35$ at $W=3.5 \mathrm{GeV}$ and 0.8 for $W \geq 5 \mathrm{GeV}$. (Color online)

$H_{T}$ by changing its normalization: $N_{H_{T}}^{u}=1.1$ and $N_{H_{T}}^{d}=-0.3$ and take for the slope $b_{H_{T}}=0.3 \mathrm{GeV}^{-2}$ (see Tab. 1). In order to have more or less the same $\pi^{0}$ cross section at CLAS kinematics the GPD $\bar{E}_{T}^{a}$ is multiplied by a factor 0.8. For this modification of the transversity GPDs our fit to $\pi^{+}$ production cross section practically remains unchanged. It is of the same quality as for the standard parameterization since $H_{T}^{u}-H_{T}^{d}$ is almost unaltered. On the other hand, the forward limits of $H_{T}$ are now at variance with the transversity PDFs given in [29] and the lowest moments of $\bar{E}_{T}$ are below the lattice-QCD results [14]. As one sees from Fig. 6 the dip at $t^{\prime}$ is considerably less deep now. The longitudinal-transverse and transverse-transverse interference cross sections are also shown for the standard parameterization in this figure. The analogous predictions for CLAS kinematics are in fair agreement with experiment [47]. The energy and $Q^{2}$ dependencies of the $\pi^{0}$ cross section are shown in Figs. 7.

Predictions for the $\pi^{0}$ target asymmetries are shown in Fig. 8 for the standard kinematics as an example. The transverse target asymmetries behave differently for $\pi^{0}$ and $\pi^{+}$production. Particularly noteworthy is the fact the $\sin \phi_{s}$ moment for $\pi^{0}$ production is much smaller than for $\pi^{+}$for non-zero $t^{\prime}$. This behavior can be traced back to the amplitude $\mathcal{M}_{0+,++}$ which is very small for $\pi^{+}$but large for $\pi^{0}$ production. In terms of helicity amplitudes the 


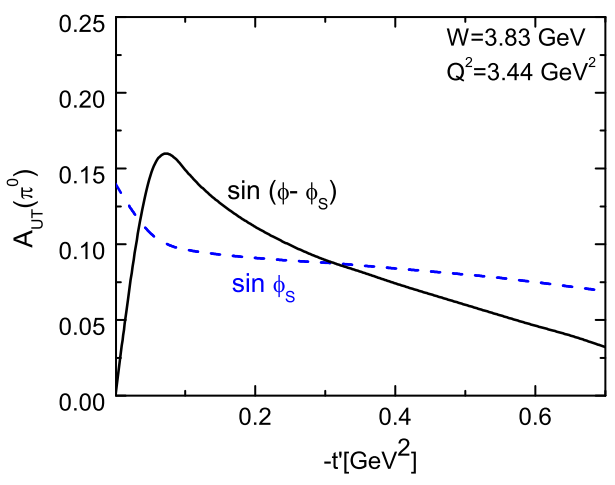

Figure 8: Results for the $\sin \left(\phi-\phi_{s}\right)$ and $\sin \phi_{s}$ moments of the transverse target asymmetries for $\pi^{0}$ electroproduction versus $t^{\prime}$. (Color online)

asymmetry reads

$$
A_{U T}^{\sin \phi_{s}} \sigma_{0} \simeq \sqrt{\varepsilon(1+\varepsilon)} \operatorname{Im}\left[\mathcal{M}_{0+,++}^{*} \mathcal{M}_{0-, 0+}-\mathcal{M}_{0-,++}^{*} \mathcal{M}_{0+, 0+}\right],
$$

where it is assumed that the angle of the rotation in the lepton plane from the direction of the incoming lepton to the virtual photon is small. The quantity $\sigma_{0}$ is given by

$$
\begin{aligned}
\sigma_{0} & =\frac{1}{2}\left[\left|\mathcal{M}_{0+,++}\right|^{2}+\left|\mathcal{M}_{0-,-+}\right|^{2}+\left|\mathcal{M}_{0-,++}\right|^{2}+\left|\mathcal{M}_{0+,-+}\right|^{2}\right] \\
& +\epsilon\left[\left|\mathcal{M}_{0+, 0+}\right|^{2}+\left|\mathcal{M}_{0-, 0+}\right|^{2}\right]
\end{aligned}
$$

The $\pi^{+}$asymmetry is essentially generated by the second term in (33) while, for not too small $-t^{\prime}$, a strong cancellation between both the terms in (33) occur for $\pi^{0}$ production. For $t^{\prime} \rightarrow 0$ the first term vanishes $\propto t^{\prime}$.

The different behavior of the $\sin \left(\phi-\phi_{s}\right)$ moment for $\pi^{+}$and $\pi^{0}$ production (see Figs. 4 and 8 ) is also a consequence of the contribution from $\bar{E}_{T}$ being small in one but large in the other reaction. Approximately this asymmetry is given by

$$
A_{U T}^{\sin \left(\phi-\phi_{s}\right)} \sigma_{0} \simeq-2 \varepsilon \operatorname{Im}\left[\mathcal{M}_{0-, 0+}^{*} \mathcal{M}_{0+, 0+}\right]-\operatorname{Im}\left[\mathcal{M}_{0+,++}^{*} \mathcal{M}_{0-,++}\right]
$$

The second term provides a positive contribution to the $\pi^{0}$ asymmetry which overcompensates the asymptotically dominant first term provided by longitudinally polarized photons. 
Our estimates of $\pi^{0}$ electroproduction which, we repeat, strongly depend on the lattice QCD findings [14] for the moments of $\bar{E}_{T}$, are distinct from our previous estimate presented in [1] where the amplitude $\mathcal{M}_{0+,++}$ had been neglected, and from other predictions. In collinear factorization to leadingtwist accuracy only the longitudinal cross section can be calculated. The predictions for it, see e.g. [10], are of about the same size as our longitudinal cross section but much smaller than the full cross section. In [49] the dominance of the transverse cross section in $\pi^{0}$ production has also been suggested. However, our treatment of the twist-3 contribution differs from the approach advocated in [49] markedly. In the latter work the subprocess is viewed as form factors for photon-pion transitions under the action of vector and axial-vector currents. A proportionality between $\bar{E}_{T}$ and $H_{T}$ is assumed where the constants of proportionality are set by the transverse anomalous magnetic moments for which the values 0.6 for $u$ and 0.3 for $d$ quarks are utilized. This parameterization of $\bar{E}_{T}$ is not supported by lattice QCD [14] (see Tab. 2) and differs from our parameterization drastically: $\bar{E}_{T}$ in [49] is much smaller than our one and has opposite signs for $u$ and $d$ quarks. As a consequence the absolute value of the amplitude $\mathcal{M}_{0-,++}$ is much larger than that of $\mathcal{M}_{0+,++}$ as opposed to our results. The results for the $\pi^{0}$ cross section are therefore substantially smaller in [49]. Instead of a forward dip a forward maximum occurs.

\section{$5 \quad$ Electroproduction of of $\eta$ and $\eta^{\prime}$}

The treatment of the $\eta$ and $\eta^{\prime}$ mesons within the handbag approach is similar to the case of the $\pi^{0}$. It is only a bit more intricate due to $\eta-\eta^{\prime}$ mixing and, in principle, the gluon-gluon Fock component of the $\eta$ and $\eta^{\prime}$ mesons. This Fock component contributes to the same order of $\alpha_{\mathrm{s}}$ as the quark-antiquark components as can be seen from the Feynman graph shown in Fig. 9. It has however been shown in [50] that the twist-2 subprocess amplitude for the gluon-gluon component is $\propto t / Q^{2}$ and is therefore to be neglected for consistency. A possible twist-3 gluon contribution is neglected as well since, in contrast to the quark subprocess (cf. (9)), there is no known reason why this contribution could be large.

Working in the flavor octet-singlet basis and exploiting the results of the quark-flavor mixing scheme developed in [51], we can decompose any of the 


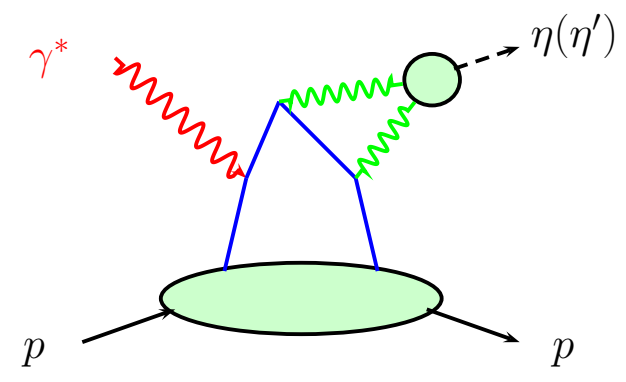

Figure 9: A typical Feynman graph for the contribution from the gluon-gluon Fock component of the $\eta$ and $\eta^{\prime}$ mesons.

helicity amplitudes as

$$
\begin{aligned}
& \mathcal{M}^{\eta}=\cos \theta_{8} \mathcal{M}^{(8)}-\sin \theta_{1} \mathcal{M}^{(1)} \\
& \mathcal{M}^{\eta^{\prime}}=\sin \theta_{8} \mathcal{M}^{(8)}+\cos \theta_{1} \mathcal{M}^{(1)}
\end{aligned}
$$

For the mixing angles we adopt the values

$$
\theta_{8}=-21.2^{\circ}, \quad \theta_{1}=-9.2^{\circ}
$$

derived in [51] on exploiting the divergencies of the axial vector current which embodies the axial-vector anomaly. The octet and singlet amplitudes are to be calculated in full analogy to the case of the $\pi^{0}$ with octet and singlet wave functions and decay constants taken from [51]

$$
f_{8}=1.26 f_{\pi}, \quad f_{1}=1.17 f_{\pi} .
$$

For these wave functions, assumed to be independent on the meson, we take the same Gaussian as for the pion with exactly the same transverse size parameters. For $Q^{2}$ in the range of interest in this work these simple wave functions suffice, cf. the remark in Sect. 4. In the analysis of the Babar data on the $P \gamma$ transition form factors [52], also performed within the modified perturbative approach [43], only little differences between the octet and singlet wave functions have been found. The GPDs appear in the following flavor combinations

$$
\begin{aligned}
& F_{i}^{(8)}=\frac{1}{\sqrt{6}}\left[e_{u} F_{i}^{u}+e_{d} F_{i}^{d}-2 e_{s} F_{i}^{s}\right] \\
& F_{i}^{(1)}=\frac{1}{\sqrt{3}}\left[e_{u} F_{i}^{u}+e_{d} F_{i}^{d}+e_{s} F_{i}^{s}\right] .
\end{aligned}
$$



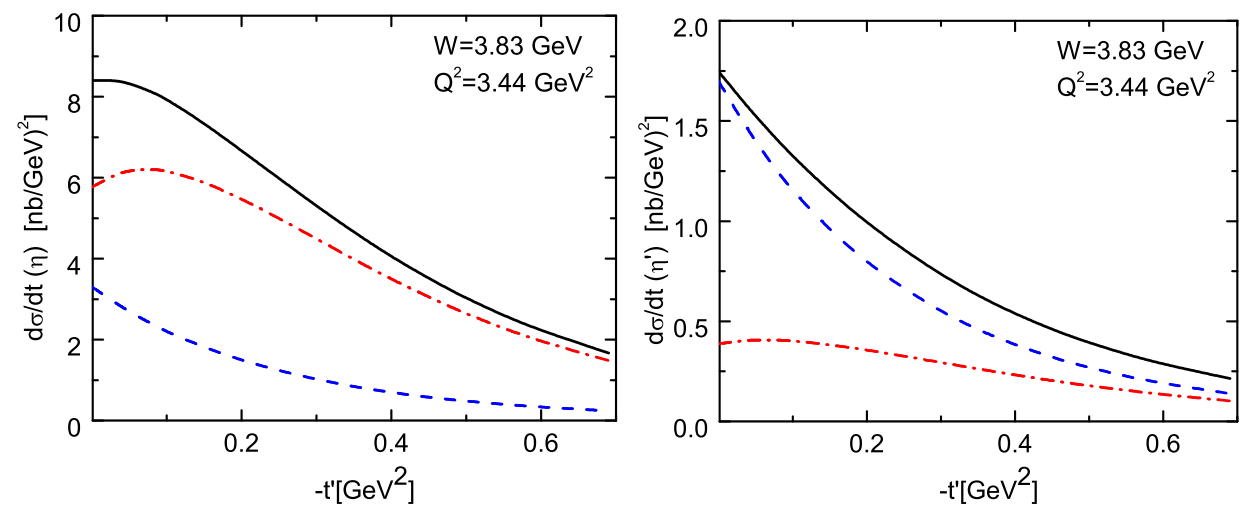

Figure 10: Results for the $\eta$ (left) and $\eta^{\prime}$ (right) cross sections. For notations refer to Fig. 5. (Color online)

For the charge-conjugation-even mesons the GPDs contribute in the valencequark combination $F_{i}^{a}-F_{i}^{\bar{a}}$. For strange quarks we assume $F_{i}^{s} \simeq F_{i}^{\bar{s}}$ for all GPDs ${ }^{7}$. Hence, there is no contribution from strange quarks and one arrives at the relation $F_{i}^{(1)}=\sqrt{2} F_{i}^{(8)}$.

The $\eta$ and $\eta^{\prime}$ cross sections are shown in Fig. 10. In order to facilitate comparison of the cross sections for the various mesons we use the same kinematical setting, $W=3.83 \mathrm{GeV}$ and $Q^{2}=3.44 \mathrm{GeV}^{2}$, for which there are $\pi^{+}$data from HERMES [5]. For an understanding of the differences between the $\eta$ and $\pi^{0}$ cross sections it is important to realize that the $u$ and $d$-quark GPDs contribute with opposite signs in these two reactions (see (39) and $(32))$. For small $-t^{\prime}$, the region where the GPDs $\widetilde{H}$ and $H_{T}$ dominate, the opposite sign of their $u$ and $d$-quark parts leads to a larger contribution to $\eta$ than to $\pi^{0}$ production which, in collaboration with the larger decay constant (38), overcompensates the relative factor $1 / \sqrt{3}$ between (39) and (32). Hence, the $\eta / \pi^{0}$ ratio of the cross sections is of order 1 , see Fig. 11. For large $-t^{\prime}$ the GPD $\bar{E}_{T}$ dominates which has the same sign for $u$ and $d$ quarks. Therefore, the $\eta / \pi^{0}$ ratio is much smaller than 1 ; in fact close to $1 / 3$ for a large range of $t^{\prime}$. Interestingly the CLAS collaboration [47] has measured the $\eta / \pi^{0}$ cross section ratio for $-t$ reaching from 0.14 to $0.77 \mathrm{GeV}^{2}$ but for

\footnotetext{
${ }^{7}$ For the unpolarized strange quark PDFs [53] and for $H^{s}$ [54] possible differences between strange and antistrange distributions have been studied. No evidence for a nonzero difference has been found within errors.
} 


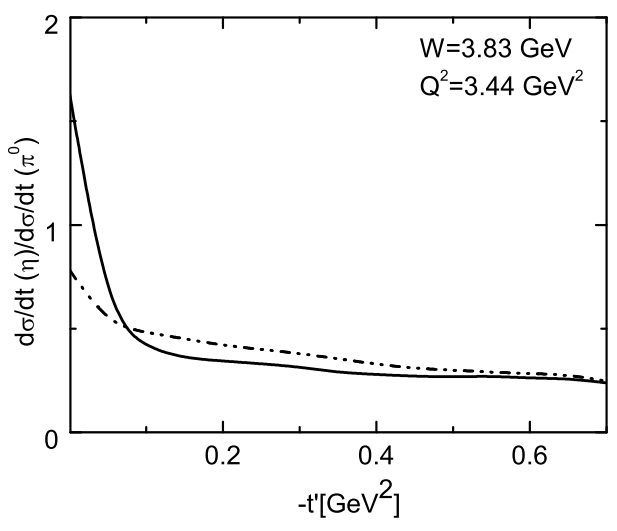

Figure 11: Ratio of the $\eta$ and $\pi^{0}$ cross sections. Solid (dash-dot-dotted) line represents the results evaluated from the standard parameterization of the GPDs (modified $H_{T}$ ). For notations refer to Fig. 5. (Color online)

values of $Q^{2}$ and $W$ that are somewhat too low for allowing a comparison with our results without reservation. Nevertheless the preliminary CLAS data match very well our predictions which can be regarded as an indication of large contributions from the GPD $\bar{E}_{T}$ with the same sign for its $u$ and $d$-quark parts. We note in passing that our $\eta / \pi^{0}$ ratio of the longitudinal cross sections at low $-t^{\prime}$ is in agreement with an estimate presented in [55].

The transverse cross section for $\eta^{\prime}$ production is much smaller than those for the $\eta$ and $\pi^{0}$ channels. The reason is obvious. The twist- 3 mechanism is not enhanced by the chiral condensate for the flavor-singlet part. Actually it is about half as strong as for the octet channels.

In Fig. 12 the $\sin \left(\phi-\phi_{s}\right)$ and the $\sin \phi_{s}$ moments of the $\eta$ and $\eta^{\prime}$ cross sections measured with a transversely polarized target are shown. The trends of these asymmetries for $\eta$ production bear similarities to the corresponding $\pi^{0}$ ones while for the $\eta^{\prime}$ the suppression of the twist-3 effect reflects itself in a different behavior of the asymmetries.

\section{The kaon-hyperon channels}

The analysis of kaon electroproduction is similar to $\pi^{+}$production; the same expressions hold for the convolutions $\left(e_{d}=e_{s}\right)$. To describe the $K$-meson we again use a Gaussian wave function with a transverse-size parameter $a_{K}=a_{\pi}$ 

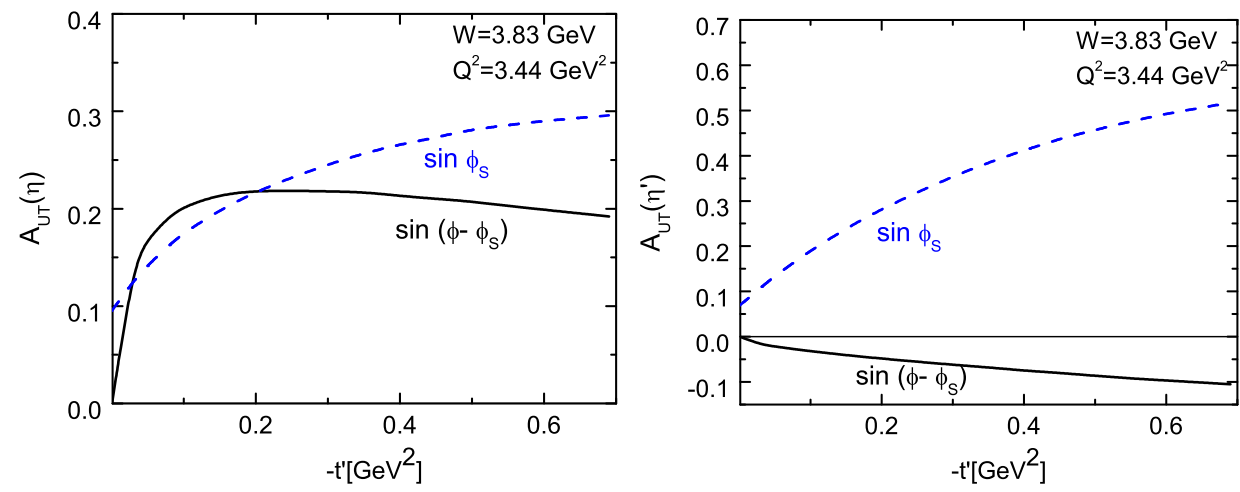

Figure 12: Results for the $\sin \left(\phi-\phi_{s}\right)$ and $\sin \phi_{s}$ moments of the transverse target asymmetries for $\eta$ (left) and $\eta^{\prime}$ (right) electroproduction versus $t^{\prime}$. (Color online)

and associated distribution amplitudes, the flat one at twist-3 level [56] and

$$
\Phi_{K}(\tau, \mu)=\Phi_{A S}(\tau)\left[1+a_{K 1}(\mu) C_{1}^{3 / 2}(2 \tau-1)+a_{K 2}(\mu) C_{2}^{3 / 2}(2 \tau-1)+\ldots\right]
$$

for twist 2 (the momentum fraction $\tau$ refers to the $u$ quark). The important difference to the case of pions is that this distribution amplitude is not symmetric in the momentum fractions $\tau$ and $1-\tau$ in general. A restriction to the asymptotic distribution amplitude is inappropriate for kaons since it would lead to the relation

$$
\mathcal{H}^{K}=f_{K} / f_{\pi} \mathcal{H}^{\pi}
$$

among the twist-2 subprocess amplitudes. With $f_{K}=1.21 f_{\pi}$ the kaon amplitudes would then be larger than the pion ones. This is against experience; kaon channels are typically suppressed by about $10 \%$ against pion channels. This can be seen, for instance, in the electromagnetic form factors [57], in two-photon annihilations [58] or $\chi_{c J}$ decays into pairs of kaons or pions [59]. For the lowest two Gegenbauer coefficients we use the values

$$
a_{K 1}(1 \mathrm{GeV})=-0.1, \quad a_{K 2}(1 \mathrm{GeV})=-0.1,
$$

which are in agreement with the $\chi_{c J} \rightarrow K \bar{K}$ decay width $(J=0,2)$ [60]. QCD sum rules [56], on the other hand, provide $a_{K 1}=-0.06 \pm 0.03$ and 
$a_{K 2}=0.30 \pm 0.15$. The negative values of $a_{K 1}$ imply that, as expected, the antistrange quark in the kaon carries a larger momentum fraction on the average than the non-strange one $\left(\langle\tau\rangle=1 / 2+3 / 10 a_{K 1} ;\langle 1-\tau\rangle=\right.$ $\left.1 / 2-3 / 10 a_{K 1}\right)$. We take the first two Gegenbauer terms with the coefficients (42), into account in order to avoid an overestimate of kaon production. Their suppression within the modified perturbative approach (cf. the discussion in Sect. 4) is mild. For the twist-3 distribution amplitude we ignore such an effect.

For kaon production the proton-hyperon transition GPDs enter which, with the help of $\mathrm{SU}(3)$ flavor symmetry, can be related to the proton GPDs by $[9]$

$$
\begin{aligned}
F_{i p \rightarrow \Lambda} & \simeq-\frac{1}{\sqrt{6}}\left[2 F_{i}^{u}-F_{i}^{d}-F_{i}^{s}\right], \\
F_{i p \rightarrow \Sigma^{0}} & \simeq-\frac{1}{\sqrt{2}}\left[F_{i}^{d}-F_{i}^{s}\right] \\
F_{i p \rightarrow \Sigma^{+}} & \simeq-F_{i}^{d}+F_{i}^{s},
\end{aligned}
$$

As for the other channels we assume a flavor-symmetric sea for all GPDs and $F_{i}^{s}-F_{i}^{\bar{s}} \simeq 0$. In contrast to the case of the $\pi^{+}$where the transition GPDs $F_{i p \rightarrow n}$ are related to the proton ones by isospin invariance, one has to be aware of possible large flavor-symmetry breaking effects in (43).

Finally, we have to discuss the kaon pole. It is treated in analogy to the pion pole (see left hand graph in Fig. 1). The contribution of the kaon pole to $\widetilde{E}_{p \rightarrow Y}$ is given in (20) and that to the $p \rightarrow Y$ form factor reads

$$
F_{K Y}^{\text {pole }}(t)=\frac{(m+M) f_{K} g_{K p Y}}{m_{K}^{2}-t} F_{K p Y}(t),
$$

where the form factor $F_{K p Y}$ parameterizes a residual $t$ dependence like the one which occurs for the pion. For this form factor which has not been considered in previous work (see for instance [61]), one may take the same expression as for the case of the pion (23) for a first estimate. The GPD $\widetilde{E}$ satisfies the sum rule [61]

$$
\int_{-1}^{1} d x \widetilde{E}_{p \rightarrow Y}(x, \xi, t)=F_{K Y}(t)+\frac{1}{\xi} g_{2}^{K Y}(t) .
$$

Due to violation of flavor symmetry by the strange quark mass the extra form factor $g_{2}^{K Y}$ occurs in the sum rule. This of no relevance here since the sum 
rule (45) will not be exploited. It is important to realize that in (20) and (45) the transition GPD actually appears, no assumption on flavor symmetry is required. For the kaon-baryon coupling constants appearing in (44) we use values obtained from fixed-t dispersion relations which are however in good agreement with $\mathrm{SU}(3)$ predictions [62]

$$
g_{K^{+} p \Lambda} \simeq-13.3, \quad g_{K^{+} p \Sigma^{0}}=-g_{K^{0} p \Sigma^{+}} / \sqrt{2} \simeq-3.5 .
$$

The relation between the coupling constants for $K^{+} p \Sigma^{0}$ and $K^{0} p \Sigma^{+}$is a consequence of isospin invariance. The residue of the kaon pole (5) is fully fixed by noting that we take for the electromagnetic form factor of the positively charged kaon that of the pion but multiplied by a factor of 0.9 in order to take care of flavor symmetry breaking

$$
F_{K^{+}}\left(Q^{2}\right)=\frac{0.9}{1+Q^{2} / 0.462 \mathrm{GeV}^{2}} .
$$

This is at least in agreement with the CLEO measurement [57] of the timelike pion and kaon electromagnetic form factors.

The electromagnetic form factor of the $K^{0}$ is not necessarily zero. In the perturbative frame work for instance it is related to the antisymmetric Gegenbauer coefficients in (40). Since nothing is known about this form factor we assume its ratio to the $K^{+}$form factor to be given by the perturbative ratio

$$
F_{K^{0}} / F_{K^{+}} \approx \frac{4}{3} \frac{a_{K 1}\left(1+a_{K 2}\right)}{1+a_{K 1}^{2}+a_{K 2}^{2}+2 a_{K 2}} .
$$

Evaluating this ratio from the Gegenbauer coefficient quoted in (42), one finds a value of -0.15 .

Our results for cross sections and target asymmetries are shown in Figs. 13 and 14. The kaon cross sections are dominated by contributions from transversely polarized photons too. As opposed to $\pi^{+}$production the mesonpole term plays a minor role here because of the large kaon mass appearing in the pole denominator. It is however strong enough to produce a little maximum in the longitudinal cross section for $K^{+} \Lambda$ at $t^{\prime} \simeq-0.1 \mathrm{GeV}^{2}$ by means of the factor $-t /\left(t-m_{K}^{2}\right)^{2}$ in the pure pole contribution $\left(m_{K}^{2}>-t_{0}\right)$. For the two other kaon channels the pole contribution is hardly visible. The cross section for the $K^{+} \Lambda$ channel is substantially larger than those for the other kaon channels. Besides different pole contributions, these two processes differ from each other by the factor $1 / \sqrt{2}$ in the GPD combinations (43) in 

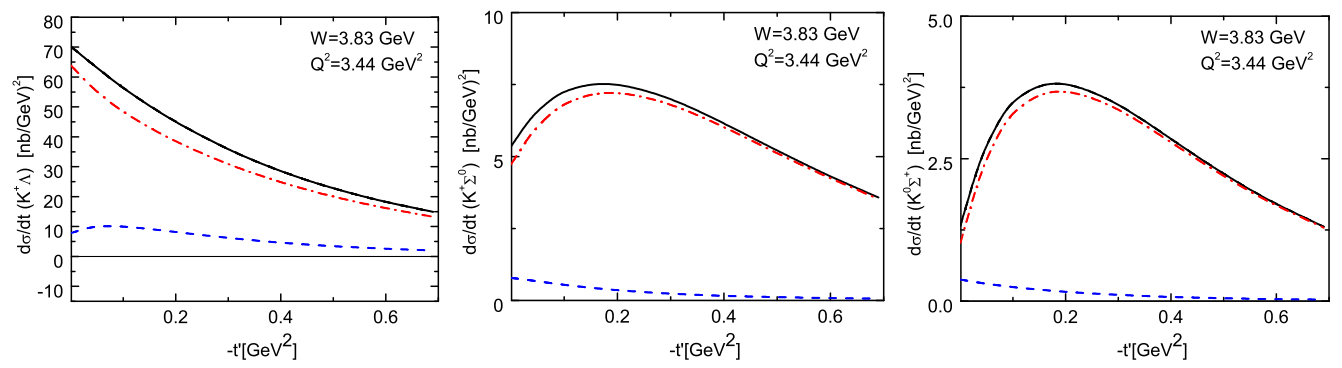

Figure 13: Results for the cross sectios of the $K^{+} \Lambda$ (left), $K^{+} \Sigma^{0}$ (center) and $K^{0} \Sigma^{+}$(right) final states. For notations refer to Fig. 5. (Color online)
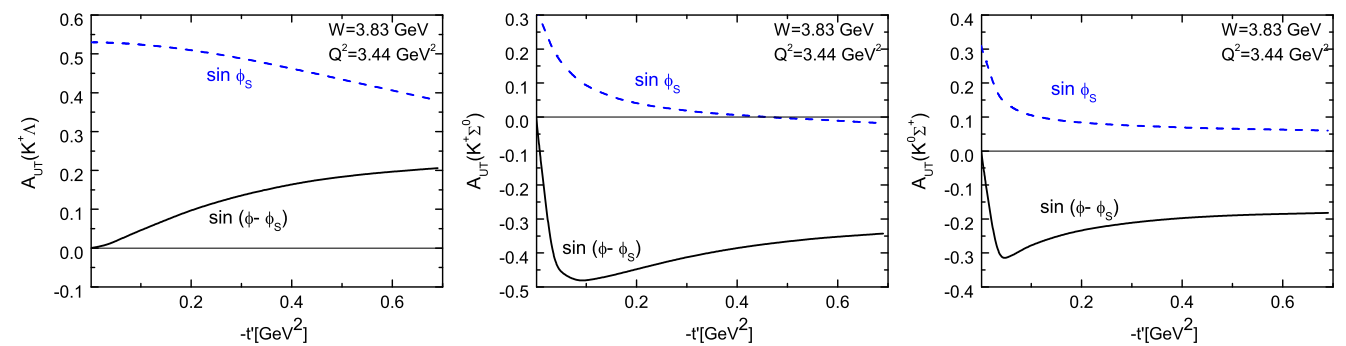

Figure 14: Results for the $\sin \left(\phi-\phi_{s}\right)$ and $\sin \phi_{s}$ moments of the transverse target asymmetries for electroproduction of the $K^{+} \Lambda$ (left), $K^{+} \Sigma^{0}$ (center) and $K^{0} \Sigma^{+}$(right) final states versus $t^{\prime}$. (Color online)

favor of $\gamma^{*} p \rightarrow K^{0} \Sigma^{+}$. This factor is overcompensated by the $u$-quark charge in the hard amplitude of the process $\gamma^{*} p \rightarrow K^{+} \Sigma^{0}$ instead of $e_{d}$ in the other process. As a result the $K^{+} \Sigma^{0}$ cross section is about twice as large as the $K^{0} \Sigma^{+}$one. On the other hand, the transverse target asymmetries behave very similar for both the processes, see Fig. 14. The asymmetries for the $K^{+} \Lambda$ channel are similar to those for $\pi^{+}$production.

Finally, in Fig. 15, we compare the various cross sections at a larger value of $W$, characteristic of the COMPASS experiment. 


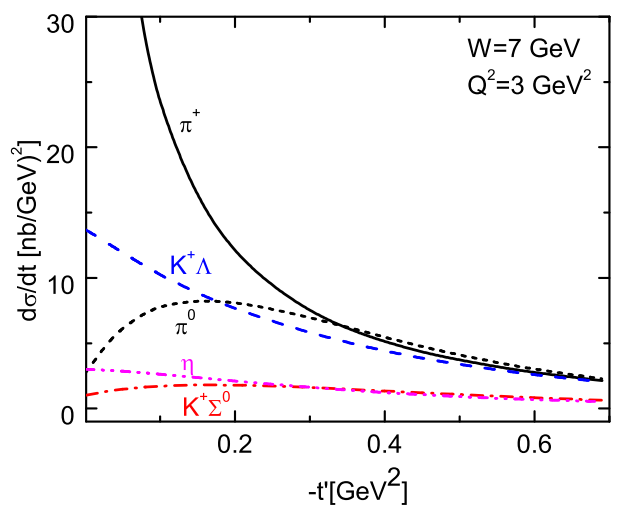

Figure 15: The cross sections for various pseudoscalar meson channels at $W=7 \mathrm{GeV}$ and $Q^{2}=3 \mathrm{GeV}^{2}$. (Color online)

\section{The longitudinal beam and target asymmetries}

In Fig. 16 we display the beam-spin asymmetry for the pseudoscalar-meson channels at the standard kinematical setting. In terms of helicity amplitudes this observable is expressed by

$$
\begin{aligned}
A_{L U} \sigma_{0} & =\sqrt{\varepsilon(1-\varepsilon)} \operatorname{Im}\left[\left(\mathcal{M}_{0+,++}^{*}-\mathcal{M}_{0+,-+}^{*}\right) \mathcal{M}_{0+, 0+}\right. \\
& \left.+\left(\mathcal{M}_{0-,++}^{*}-\mathcal{M}_{0-,-+}^{*}\right) \mathcal{M}_{0-, 0+}\right]
\end{aligned}
$$

In our handbag approach the first term in (49) cancels (see (11)) and the second one simplifies to

$$
A_{L U} \sigma_{0} \simeq \sqrt{\varepsilon(1-\varepsilon)} \operatorname{Im}\left[\mathcal{M}_{0-,++}^{*} \mathcal{M}_{0-, 0+}\right] .
$$

For reactions being dominated by $\bar{E}_{T}$ as for example $\pi^{0}$ production, $A_{L U}$ is very small. Others like $\pi^{+}$production for which $\bar{E}_{T}$ is less prominent, are large. This can be clearly seen in Fig. 16. The CLAS collaboration [63] has measured $A_{L U}$ for $\pi^{0}$ and $\pi^{+}$production at $W \simeq 2.4 \mathrm{GeV}$ and $Q^{2} \simeq 2.5 \mathrm{GeV}^{2}$. While the CLAS result for $\pi^{+}$production is in reasonable agreement with our result, is that for $\pi^{0}$ production larger than our one.

Another interesting observable is the $\sin \phi$-moment of the electroproduction cross section measured with a longitudinally polarized target. To the 

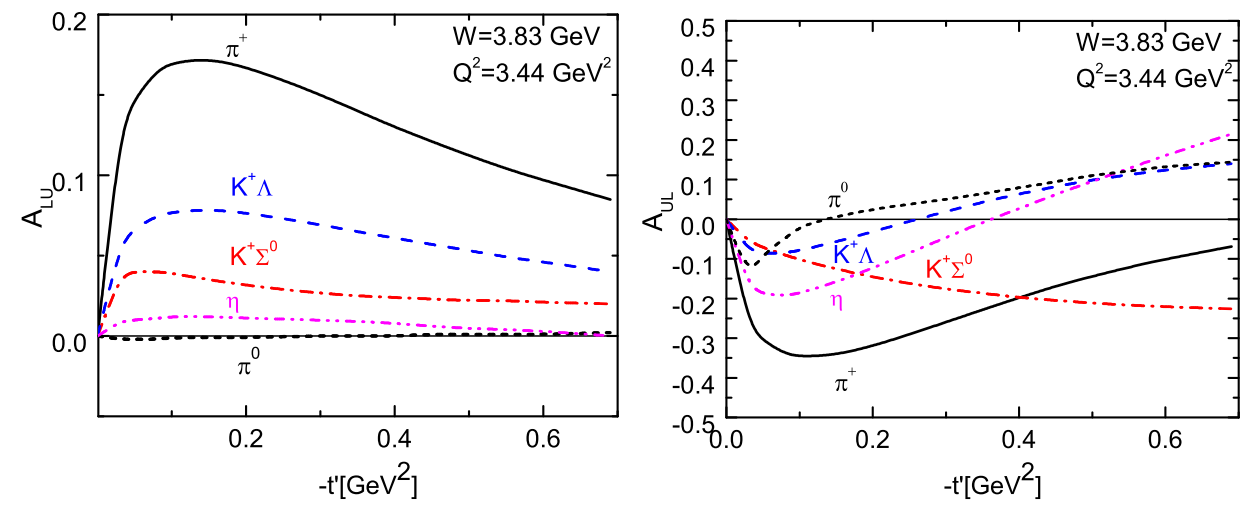

Figure 16: The beam spin asymmetry (left) and the asymmetry for a longitudinally polarized target (right) for various pseudoscalar-meson channels versus $t^{\prime}$. (Color online)

extend that the angle of the rotation in the lepton plane from the direction of the incoming lepton to the virtual photon is small the longitudinal target asymmetry reads

$$
\begin{gathered}
A_{U L} \sigma_{0} \simeq-\sqrt{\varepsilon(1+\varepsilon)} \operatorname{Im}\left[\left(\mathcal{M}_{0+,++}^{*}+\mathcal{M}_{0+,-+}^{*}\right) \mathcal{M}_{0+, 0+}\right. \\
\left.+\left(\mathcal{M}_{0-,++}^{*}+\mathcal{M}_{0-,-+}^{*}\right) \mathcal{M}_{0-, 0+}\right] .
\end{gathered}
$$

It simplifies in our handbag approach to

$$
\begin{gathered}
A_{U L} \sigma_{0} \simeq-\sqrt{\varepsilon(1+\varepsilon)} \operatorname{Im}\left[2 \mathcal{M}_{0+,++}^{*} \mathcal{M}_{0+, 0+}\right. \\
\left.+\mathcal{M}_{0-,++}^{*} \mathcal{M}_{0-, 0+}\right] .
\end{gathered}
$$

Without the GPD $\bar{E}_{T}$ the ratio of the two asymmetries would be given by $A_{U L} / A_{L U}=-\sqrt{(1+\varepsilon) /(1-\varepsilon)}$. Deviations from this ratio signal a non-zero contributions from $\bar{E}_{T}$, see Fig. 16 .

\section{Summary}

We have investigated electroproduction of pseudoscalar mesons within the handbag approach restricting ourselves to the range of small skewness. Forced 
by the behavior of target asymmetries for $\pi^{+}$production measured by the HERMES collaboration [6] we have allowed for a twist-3 effect consisting of a twist-3 meson distribution amplitude in combination with the leading-twist transversity GPDs. This twist-3 effect is strongly enhanced by the chiral condensate (9), (10). It therefore seems to be justified to include this effect in the analysis even though we don't perform a systematic analysis at twist-3 accuracy. We stress that for electroproduction of vector mesons the twist-3 effect is expected to be much smaller since $\mu_{P}$ is to be replaced by the vector meson's mass.

The GPDs are constructed from double distributions which are parameterized as zero-skewness GPDs multiplied by a Regge-like $t$ dependence and a weight function from which the skewness dependence of the GPD is generated. They are constrained by form factors and PDFs. Their isovector combination are fitted to the HERMES data on $\pi^{+}$electroproduction. Moments of the GPDs are compared to moments obtained from lattice QCD. In general reasonable agreement is to be observed. A number of positivity bounds for the GPDs are checked as well by us. An exceptional case is $\bar{E}_{T}$. Its parameterization is fully constrained by lattice-QCD results [14] since this is the only available information about it at present. Therefore, $\bar{E}_{T}$ suffers from the usual uncertainties of lattice-QCD results arising from the still low statistics and from the compulsion of working with heavy quarks. It turns out that the GPD $\bar{E}_{T}$ for $u$ and $d$ quarks cancel to a high degree in $\pi^{+}$electroproduction while it plays a prominent role in most of the other pseudoscalar-meson channels. In fact, with the exception of $\pi^{+}$and $\eta^{\prime}$ they are all dominated by contributions from the transversity GPDs, i.e. by transversely polarized photons, in the range of $Q^{2}$ accessible in present-day experiments. Of course, for $Q^{2} \rightarrow \infty$ the longitudinal cross sections will dominate in all reactions. These findings are to be contrasted with the usual assumption made in the handbag approach [9]-[12] that contributions from longitudinally polarized photons already dominate for $Q^{2}$ of the order of a few $\mathrm{GeV}$.

A precise calculation of all details of electroproduction of pseudoscalar mesons is beyond feasibility at present. There are many uncertainties like the parameterization of the transversity GPDs or the exact treatment of the twist-3 contribution (e.g. the neglect of possible three-particle configuration of the meson state). Also higher-order perturbative corrections other than those included in the Sudakov factor and, implicitly, in the experimental electromagnetic form factors of the mesons, are ignored. According to [64] the NLO corrections are rather large for the cross sections for $Q^{2} \lesssim 10 \mathrm{GeV}$. 
However, the results presented in [64] refer to the leading-twist contribution to $\pi^{+}$production, i.e. to about $10 \%$ of the cross section since only the perturbative contribution to the pion form factor is taken into account. Thus, it is not clear how strong the NLO corrections are in our approach. Further uncertainties occur for kaon production. In contrast to the case of the $\pi^{+}$ where the $p \rightarrow n$ transition GPDs are related to the diagonal proton ones by isospin symmetry, the proton - hyperon transition GPDs are connected to the proton GPDs by $\mathrm{SU}(3)$ flavor symmetry which is less accurate than isospin symmetry. The assumption of a flavor symmetric sea for all GPDs is also stronger for kaons than for pions. With regard to all these uncertainties we consider our investigation of electroproduction of pseudoscalar mesons as an estimate of the pertinent observables. The trends and magnitudes of the cross sections are likely correct but probably not the details. Particularly large uncertainties are to be expected for subtle observables as the spin asymmetries. On the other hand, the large magnitude of contributions from the transversity GPDs seems to us hard to avoid. Future data measured at the upgraded Jefferson Lab or by the COMPASS collaboration may probe our results. Although our approach is designed for small skewness (and rather large $Q^{2}$ and $W$ ) we also compared our results with the large skewness data measured at the present JLab accelerator. We found general agreement in trends and magnitudes. We regard this as a hint at strong contributions from the transversity GPD $\bar{E}_{T}$ for which the $u$ and $d$ quark parts have the same sign.

Acknowledgements We thank Ph. Hägler for drawing our attention to [14]. We are also grateful to H. Avagyan, M. Diehl, V. Kubarowsky, A. Metz and W.-D. Nowak for valuable discussions. This work is supported in part by the Russian Foundation for Basic Research, Grant 09-02-01149 and by the Heisenberg-Landau program and by the BMBF, contract number 06RY258.

\section{References}

[1] S. V. Goloskokov and P. Kroll, Eur. Phys. J. C 65, 137 (2010) [arXiv:0906.0460 [hep-ph]].

[2] S. V. Goloskokov and P. Kroll, Eur. Phys. J. C 50, 829 (2007) [arXiv:hep-ph/0611290]; 
[3] S. V. Goloskokov and P. Kroll, Eur. Phys. J. C 53, 367 (2008) [arXiv:0708.3569 [hep-ph]].

[4] J. Botts and G. Sterman, Nucl. Phys. B 325, 62 (1989).

[5] A. Airapetian et al. [HERMES Collaboration], Phys. Lett. B 659, 486 (2008) [arXiv:0707.0222 [hep-ex]].

[6] A. Airapetian et al. [HERMES Collaboration], Phys. Lett. B 682, 345 (2010) [arXiv:0907.2596 [hep-ex]].

[7] A. Airapetian et al [HERMES Collaboration], Phys. Lett. B535, 85 (2002).

[8] L. Mankiewicz, G. Piller and T. Weigl, Eur. Phys. J. C5, 119 (1998).

[9] L. L. Frankfurt, P. V. Pobylitsa, M. V. Polyakov and M. Strikman, Phys. Rev. D 60, 014010 (1999) [arXiv:hep-ph/9901429].

[10] K. Goeke, M. V. Polyakov and M. Vanderhaeghen, Progr. Part. Nucl. Phys. Vol. 47, No. 2 (2001) [arXiv:hep-ph/0106012].

[11] M. Diehl, W. Kugler, A. Schafer and C. Weiss, Phys. Rev. D 72, 034034 (2005) [Erratum-ibid. D 72, 059902 (2005)] [arXiv:hep-ph/0506171].

[12] C. Bechler and D. Mueller, arXiv:0906.2571 [hep-ph].

[13] H. P. Blok et al. [Jefferson Lab Collaboration], Phys. Rev. C 78, 045202 (2008) [arXiv:0809.3161 [nucl-ex]].

[14] M. Gockeler et al. [QCDSF Collaboration and UKQCD Collaboration], Phys. Rev. Lett. 98, 222001 (2007) [arXiv:hep-lat/0612032].

[15] P. Hoodbhoy and X. Ji, Phys. Rev. D58, 054006 (1998) [hep$\mathrm{ph} / 9801369]$.

[16] M. Diehl, Eur. Phys. J. C19, 485 (2001) [hep-ph/0101335].

[17] E. Leader, A. V. Sidorov and D. B. Stamenov, arXiv:1103.5979 [hep-ph].

[18] D. de Florian, R. Sassot, M. Stratmann and W. Vogelsang, Phys. Rev. D 80, 034030 (2009) [arXiv:0904.3821 [hep-ph]]. 
[19] I. V. Musatov and A. V. Radyushkin, Phys. Rev. D 61, 074027 (2000) [hep-ph/9905376].

[20] D. Mueller et al., Fortsch. Phys. 42, 101 (1994) [hep-ph/9812448].

[21] A. V. Radyushkin, Phys. Lett. B 449, 81 (1999) [hep-ph/9810466].

[22] A. Boyarski et al, Phys. Rev. Lett. 20, 300 (1968).

[23] J. Blümlein and H. Böttcher, Nucl. Phys. B 636, 225 (2002) [hep$\mathrm{ph} / 0203155]$

[24] M. Diehl, T. Feldmann, R. Jakob and P. Kroll, Eur. Phys. J. C 39, 1 (2005) [hep-ph/0408173].

[25] T. Kitagaki et al, Phys. Rev. D28, 436 (1983).

[26] Ph. Hagler et al. [LHPC Collaborations], Phys. Rev. D 77, 094502 (2008) [arXiv:0705.4295 [hep-lat]].

[27] S. Choi et al, Phys. Rev. Lett. 71, 3927 (1993).

[28] C. Hanhart, AIP Conf. Proc. 1322, 169 (2010) [arXiv:1009.5226 [nuclth]].

[29] M. Anselmino, M. Boglione, U. D’Alesio, A. Kotzinian, F. Murgia, A. Prokudin and S. Melis, Nucl. Phys. Proc. Suppl. 191, 98 (2009) [arXiv:0812.4366 [hep-ph]].

[30] J. Pumplin, D. R. Stump, J. Huston, H. L. Lai, P. M. Nadolsky and W. K. Tung, JHEP 0207 (2002) 012 [arXiv:hep-ph/0201195].

[31] M. Gockeler et al. [QCDSF Collaboration and UKQCD Collaboration], Phys. Lett. B 627, 113 (2005) [arXiv:hep-lat/0507001].

[32] T. Ledwig, A. Silva and H. C. Kim, Phys. Rev. D 82, 034022 (2010) [arXiv:1004.3612 [hep-ph]].

[33] M. Burkardt, AIP Conf. Proc. 915, 313 (2007) [arXiv:hep-ph/0611256].

[34] B. Pasquini et al, Phys. Rev. D 72, 094029 (2005) [hep-ph/0510376]. 
[35] D. Boer, P. J. Mulders, Phys. Rev. D57, 5780-5786 (1998). [hep$\mathrm{ph} / 9711485]$.

[36] V. Barone, S. Melis, A. Prokudin, Phys. Rev. D81, 114026 (2010). [arXiv:0912.5194 [hep-ph]].

[37] A. Bressan [ COMPASS Collaboration ], [arXiv:0907.5511 [hep-ex]].

[38] F. Giordano et al. [ On behalf of the HERMES Collaboration ], AIP Conf. Proc. 1149, 423-426 (2009). [arXiv:0901.2438 [hep-ex]].

[39] M. Diehl and Ph. Hägler, Eur. Phys. J. C 44, 87 (2005) [arXiv:hep$\mathrm{ph} / 0504175]$.

[40] P. V. Pobylitsa, Phys. Rev. D65, 114015 (2002) [hep-ph/0201030].

[41] X. -d. Ji, J. -P. Ma, F. Yuan, Eur. Phys. J. C33, 75-90 (2004). [hep$\mathrm{ph} / 0304107]$.

[42] J. Bolz, P. Kroll, J. G. Korner, Z. Phys. A350, 145-159 (1994). [hep$\mathrm{ph} / 9403319]$.

[43] P. Kroll, Eur. Phys. J. C71, 1623 (2011). [arXiv:1012.3542 [hep-ph]].

[44] S. A. Morrow et al [CLAS collaboration], Eur. Phys. J. A39, 5 (2009) [arXiv:0807.3834 [hep-ex]].

[45] P. Kroll, talk given at SPIRES Conference C10/05/31, arXiv:1009.2356 [hep-ph].

[46] J. P. Santoro et al. [CLAS Collaboration], Phys. Rev. C 78, 025210 (2008) [arXiv:0803.3537 [nucl-ex]].

[47] V. Kubarovsky et al, Proceedings of the 4th Workshop " Exclusive reactions at High Momentum Transfer", Newport News, VA USA, 18-21 May 2010

[48] E. Fuchey, A. Camsonne, C. Munoz Camacho, M. Mazouz, G. Gavalian, E. Kuchina, M. Amarian, K. A. Aniol et al., Phys. Rev. C83, 025201 (2011). [arXiv:1003.2938 [nucl-ex]].

[49] S. Ahmad, G. R. Goldstein and S. Liuti, Phys. Rev. D 79, 054014 (2009) [arXiv:0805.3568 [hep-ph]]. 
[50] P. Kroll and K. Passek-Kumericki, Phys. Rev. D 67, 054017 (2003) [hep-ph/0210045].

[51] T. Feldmann, P. Kroll and B. Stech, Phys. Rev. D 58, 114006 (1998) [arXiv:hep-ph/9802409].

[52] P. del Amo Sanchez et al. [BABAR Collaboration], arXiv:1101.1142 [hep-ex].

[53] H. L. Lai, P. M. Nadolsky, J. Pumplin, D. Stump, W. K. Tung and C. P. Yuan, JHEP 0704, 089 (2007) [arXiv:hep-ph/0702268].

[54] M. Diehl, T. Feldmann and P. Kroll, Phys. Rev. D 77, 033006 (2008) [arXiv:0711.4304 [hep-ph]].

[55] M. I. Eides, L. L. Frankfurt and M. I. Strikman, Phys. Rev. D 59, 114025 (1999) [arXiv:hep-ph/9809277].

[56] P. Ball, V. M. Braun and A. Lenz, JHEP 0605, 004 (2006). [hep$\mathrm{ph} / 0603063]$.

[57] T. K. Pedlar et al. [CLEO Collaboration], Phys. Rev. Lett. 95, 261803 (2005) [arXiv:hep-ex/0510005].

[58] H. Nakazawa et al. [BELLE Collaboration], Phys. Lett. B 615 (2005) 39 [arXiv:hep-ex/0412058].

[59] K. Nakamura et al. [Particle Data Group], J. Phys. G 37, 075021 (2010).

[60] J. Bolz, P. Kroll and G. A. Schuler, Eur. Phys. J. C 2, 705 (1998) [arXiv:hep-ph/9704378].

[61] A. V. Belitsky and A. V. Radyushkin, Phys. Rept. 418, 1 (2005) [arXiv:hep-ph/0504030].

[62] O. Dumbrajs, R. Koch, H. Pilkuhn, G. c. Oades, H. Behrens, J. j. De Swart and P. Kroll, Nucl. Phys. B 216 (1983) 277.

[63] H. Avakian and L. Elouadrhiri, proceedings of the Dubna workshop (2003).

[64] M. Diehl, W. Kugler, Eur. Phys. J. C52, 933-966 (2007). [arXiv:0708.1121 [hep-ph]]. 